\title{
Evolution of the patella and patelloid in marsupial mammals
}

\author{
Alice L Denyer ${ }^{1}$, Sophie Regnault ${ }^{1,2}$, John R Hutchinson ${ }^{\text {Corresp. } 1}$ \\ ${ }^{1}$ Structure \& Motion Laboratory, Department of Comparative Biomedical Sciences, The Royal Veterinary College, North Mymms, Hertfordshire, United \\ Kingdom \\ ${ }^{2}$ Museum of Comparative Zoology and Department of Organismic and Evolutionary Biology, Harvard University, Cambridge, MA, United States \\ Corresponding Author: John R Hutchinson \\ Email address: jhutchinson@rvc.ac.uk
}

The musculoskeletal system of marsupial mammals has numerous unusual features beyond the pouch and epipubic bones. One example is the widespread absence or reduction (to a fibrous "patelloid") of the patella ("kneecap") sesamoid bone, but prior studies with coarse sampling indicated complex patterns of evolution of this absence or reduction. Here, we conducted an in-depth investigation into the form of the patella of extant marsupial species and used the assembled dataset to reconstruct the likely pattern of evolution of the marsupial patella. Critical assessment of the available literature was followed by examination and imaging of museum specimens, as well as CT scanning and histological examination of dissected wet specimens. Our results, from sampling about $19 \%$ of extant marsupial species-level diversity, include new images and descriptions of the fibrocartilaginous patelloid in Thylacinus cynocephalus (the thylacine or "marsupial wolf") and other marsupials as well as the ossified patella in Notoryctes 'marsupial moles', Caenolestes shrew opossums, bandicoots and bilbies. We found novel evidence of an ossified patella in one specimen of Macropus rufogriseus (Bennett's wallaby), with hints of similar variation in other species. It remains uncertain whether such ossifications are ontogenetic variation, unusual individual variation, pathological or otherwise, but future studies must continue to be conscious of variation in metatherian patellar sesamoid morphology. Our evolutionary reconstructions using our assembled data vary, too, depending on the reconstruction algorithm used. A maximum likelihood algorithm favours ancestral fibrocartilaginous "patelloid" for crown clade Marsupialia and independent origins of ossified patellae in extinct sparassodonts, peramelids, notoryctids and caenolestids. A maximum parsimony algorithm favours ancestral ossified patella for the clade [Marsupialia + sparassodonts] and subsequent reductions into fibrocartilage in didelphids, dasyuromorphs and diprotodonts; but this result changed to agree more with the maximum likelihood results if the character state reconstructions were ordered. Thus, there is substantial homoplasy in marsupial patellae regardless of the evolutionary 
algorithm adopted. We contend that the most plausible inference, however, is that metatherians independently ossified their patellae at least three times in their evolution. Furthermore, the variability of the patellar state we observed, even within single species (e.g. M. rufogriseus), is fascinating and warrants further investigation, especially as it hints at developmental plasticity that might have been harnessed in marsupial evolution to drive the complex patterns inferred here. 


\title{
2 Evolution of the patella and patelloid in marsupial
}

3 mammals

4

5

6

7

12

13

14

\author{
Alice L. Denyer ${ }^{1}$, Sophie Regnault ${ }^{1,2}$, John R. Hutchinson ${ }^{1}$ \\ ${ }^{1}$ Structure \& Motion Laboratory, Department of Comparative Biomedical Sciences, The Royal \\ Veterinary College, Hawkshead Lane, North Mymms, Hatfield, Hertfordshire, AL9 7TA, United \\ Kingdom \\ ${ }^{2}$ Museum of Comparative Zoology and Department of Organismic and Evolutionary Biology, \\ Harvard University, 26 Oxford Street, Cambridge, MA, USA \\ Corresponding Author: \\ John R. Hutchinson ${ }^{1}$ \\ Structure \& Motion Laboratory, Department of Comparative Biomedical Sciences, The Royal \\ Veterinary College, Hawkshead Lane, North Mymms, Hatfield, Hertfordshire, AL9 7TA, United \\ Kingdom \\ Email address: jhutchinson@rvc.ac.uk
}

\section{Abstract}

The musculoskeletal system of marsupial mammals has numerous unusual features beyond the pouch and epipubic bones. One example is the widespread absence or reduction (to a fibrous "patelloid") of the patella ("kneecap") sesamoid bone, but prior studies with coarse sampling indicated complex patterns of evolution of this absence or reduction. Here, we conducted an indepth investigation into the form of the patella of extant marsupial species and used the assembled dataset to reconstruct the likely pattern of evolution of the marsupial patella. Critical assessment of the available literature was followed by examination and imaging of museum specimens, as well as CT scanning and histological examination of dissected wet specimens. Our results, from sampling about 19\% of extant marsupial species-level diversity, include new images and descriptions of the fibrocartilaginous patelloid in Thylacinus cynocephalus (the thylacine or "marsupial wolf") and other marsupials as well as the ossified patella in Notoryctes 'marsupial moles', Caenolestes shrew opossums, bandicoots and bilbies. We found novel evidence of an ossified patella in one specimen of Macropus rufogriseus (Bennett's wallaby), with hints of similar variation in other species. It remains uncertain whether such ossifications are ontogenetic variation, unusual individual variation, pathological or otherwise, but future studies must continue to be conscious of variation in metatherian patellar sesamoid morphology. Our evolutionary reconstructions using our assembled data vary, too, depending on the reconstruction algorithm used. A maximum likelihood algorithm favours ancestral fibrocartilaginous "patelloid" for crown clade Marsupialia and independent origins of ossified patellae in extinct sparassodonts, peramelids, notoryctids and caenolestids. A maximum parsimony algorithm favours ancestral ossified patella for the clade [Marsupialia + 
43

44

45

46

47

48

49

50

51

52

53

54

55

56

57

58

59

60

61

62

63

64

65

66

67

68

69

70

71

72

73

74

75

76

77

78

79

80

81

82

83

84

85

86

87

88

sparassodonts] and subsequent reductions into fibrocartilage in didelphids, dasyuromorphs and diprotodonts; but this result changed to agree more with the maximum likelihood results if the character state reconstructions were ordered. Thus, there is substantial homoplasy in marsupial patellae regardless of the evolutionary algorithm adopted. We contend that the most plausible inference, however, is that metatherians independently ossified their patellae at least three times in their evolution. Furthermore, the variability of the patellar state we observed, even within single species (e.g. M. rufogriseus), is fascinating and warrants further investigation, especially as it hints at developmental plasticity that might have been harnessed in marsupial evolution to drive the complex patterns inferred here.

\section{Introduction}

Having diverged from the common ancestor of therian mammals during the late Jurassic period, some 160 million years ago (Bi et al., 2014; Figure 1), marsupials are a diverse and biologically fascinating group of mammals. A hallmark of marsupials is their developmental strategy: marsupials have relatively short gestation periods, after which the newborn crawls to the teat for a prolonged lactation phase, typically within a pouch (Alpin \& Archer, 1987). As a result, the embryo has delayed hindlimb development, and accelerated forelimb and cranial development (Hamrick, 1999; Sánchez-Villagra, 2002; Garland et al., 2007; Sears, 2009). Despite the constraints imposed by such as strategy (Garland et al., 2017), and geographical limitation to Australasia and South America today, the over 350 extant species of marsupials exhibit great diversity in their size, lifestyle, behaviour and anatomy (Walton and Richardson, 1989; Burgin et al., 2018; Mammal Diversity Database, 2020).

One such diverse anatomical feature is the patellar sesamoid ("kneecap" or simply patella). Sesamoids are skeletal elements found in connective tissues near joints (Vickaryous \& Olson, 2007; Abdala et al., 2019), that can modify the forces acting across it (e.g. acting as levers; Alexander \& Dimery, 1987; Fatima et al., 2019) and protect periarticular structures. In an intriguing departure from the mammalian norm amongst monotremes and placentals, many marsupials appear to lack a bony patella (Reese et al., 2001). Instead, many species possess a region of fibrocartilage within the quadriceps femoris $(\mathrm{QF})$ tendon at approximately the same anatomical location as a patella, and which can be considered an unmineralised sesamoid (Holladay et al., 1990; Reese et al., 2001; Inamassu et al., 2017; Abdala et al., 2019). This fibrocartilage pad is commonly referred to as a 'patelloid'. There is great diversity in the histological structure of the patelloid, including the size, degree of differentiation and structural orientation of the fibrocartilage (Reese et al., 2001).

A previous high-level study of mammalian patellar evolution (Samuels et al., 2017) indicated an unusual pattern of variation across marsupial families. Results suggested a single evolutionary origin of an ossified patella within Metatheria (the larger stem-based group of which marsupials are the only extant remnant), with instances of loss (to patelloid) in marsupials and later reversion in some groups. However, Samuels et al. (2017) cautioned: "inferences about the evolutionary history of the patella in Metatheria must remain tentative until further data become available". Data on the form of the patella are absent for many marsupial species or rely upon single observations or anecdotes within anatomical reports. There are also conflicting reports as to whether certain species possess a patella, partially owing to the fibrocartilage patelloid state not being recognised in all studies. 
89

90

91

92

93

94

95

96

97

98

99

100

101

102

103

104

105

106

107

108

109

110

111

112

113

114

115

116

117

118

119

120

121

122

123

124

125

126

127

128

129

130

131

132

133

Knowledge of the form of the patella in marsupials remains patchy, with several gaps waiting to be filled and incongruous statements requiring clarification. Throughout the literature, certain species or more inclusive clades have been extensively studied, for example macropods (kangaroos and kin), while others (e.g. many possums) have been poorly characterised. It is often broadly generalised that all marsupials, besides bandicoots and the bilby, possess a fibrocartilage patelloid instead of a bony patella (Reese et al., 2001; Vogelnest \& Allan, 2015; Inamassu et al., 2017). Earlier anatomical reports relied on direct observation to diagnose the presence of a bony patella (Waterhouse, 1846; Osgood, 1921; Finch et al., 1989; Haxton, 1944; Johnson \& Walton, 1989), but more recent studies have enabled better characteristation of patellar state through radiography, histology and computed tomography (CT) (Holladay et al., 1990; Reese et al., 2001; Inamassu et al., 2017; Vogelnest \& Allan, 2015).

The aims of this study are twofold. Firstly, we seek finer clarification of the form of the patella in marsupials at (and, where feasible, within) the species level. We attempt to fill gaps or inconsistencies within literature data through new observations of extant species, plus the recently extinct thylacine. Secondly, we combine literature reports and new observations, and use this finer-level dataset to perform ancestral states reconstruction of the patella to address lingering questions over its evolutionary pattern of gain, loss and possible regain. Specifically, we test the hypothesis of Samuels et al. (2017) that a bony patella was ancestral for marsupials.

\section{Materials \& Methods}

Our extensive synthesis of the literature was followed by firsthand observations and photography of osteological museum specimens, based on gaps in the data and availability of specimens for study. CT scanning or x-ray radiography of a select number of specimens was then carried out, dependent on the apparatus available, to test for the presence or absence of the patella. Additionally, a small number of frozen stored macropod specimens were available for dissection and histology of the QF tendon, described below. The combined literature and observational findings were then used to reconstruct the evolutionary polarity of the patella in marsupials, with character state codings following Samuels et al. (2017); described further below. Unfortunately, in much of the literature and museum collections it was not clear whether specimens were wildcaught, from zoos or otherwise, so we could not address this potential source of variation from normal vs. abnormal biomechanical loads.

\section{Survey of Osteological Museum Specimens}

Marsupial osteological specimens were examined for grossly visible bone or dessicated tendon/patelloid tissue. Specimens included those in collections held by the Natural History Museum (NHMUK, London), the University Museum of Zoology, Cambridge (UMZC), the Oxford University Museum of Natural History (OUMNH) and the National Museums of Scotland (NMS, Edinburgh). Specimen details can be found in Table S4 of Supplementary Text S1 (age and sex was generally unknown, so not included). Observations were only included for specimens with adequate preservation of the QF tendon (so that true absence of patella/patelloid could be discerned, versus possibility that these structures might have been cleaned off during preparation). For some specimens, the nature of the patella was unclear by visual examination

Peer] reviewing PDF | (2020:01:44905:1:1:NEW 21 Jul 2020) 
134 alone. These specimens were selected for CT scanning or radiographic imaging, as described 135 below. For other specimens, if we saw obvious cartilage amidst the QF tendon (i.e. shiny 136 texture/different colour consistent with cartilage rather than collagenous tendon material) we scored the specimen as having a patelloid. If a QF tendon was present, reasonably intact and showed no sign of a swelling or other discontinuity consistent with a patelloid, we scored the patella as absent. In cases where there was too little soft tissue remaining with the specimen (e.g. most disarticulated femur-tibia-fibula bones), we refrained from scoring that specimen. This relatively conservative approach considerably reduced our sample size but allowed for cautious scoring, and we amended the sample size problem by as comprehensive a tour through major United Kingdom zoological museum collections as we could manage within the time constraints of the study.

\section{Computed Tomography (CT) Scanning and X-Ray Radiography of Osteological Specimens} segmented using Mimics (Materialise Inc., Leuven, Belgium) software. Scan details are in Table S1 of Supplementary Text S1; patellar state coding results are in Table S5 of Supplementary Text S1. Radiographs of seven skeletal specimens from NHMUK were also obtained. Details of these radiographs are in Table S2 of Supplementary Text S1; patellar state coding results are in Table S6 of Supplementary Text S1. The CT and radiographic images were used to diagnose the presence or absence of an ossified patella within the QF tendon of the preserved specimens imaged. In the case of CT scans, it was also possible to visualise soft tissue densities (using thresholds set to cortical bone density) and therefore test for the presence of a (fibrocartilage) patelloid. For ossified patellae, the thin cortical shell and internal trabeculae typical of an ossified mammalian patella normally were visible, so scoring such specimens as "ossified" was simple. For potentially fibrocartilaginous patelloids, CT scan or X-ray data helped illuminate the composition of the QF tendon, beyond what was externally visible for the specimen. This was aided by the typical presence of the lateral sesamoid (parafibula / "fabella" / "sesamoid bone of Vesalli") proximal to the fibula (e.g. see figures in Results), as a gauge for the expected structure and densities of an ossified sesamoid. If we observed a denser, structured unit within the QF tendon in a position (proximal to the femoral condyles/tibia, in the middle of the QF tendon), we scored it as a a fibrocartilaginous patelloid. If we saw no such striking heterogeneity within the (relatively intact) QF tendon, we scored the patella as absent. Otherwise we omitted that specimen from the study. Raw scan data are available at https://figshare.com/s/c3f5c083910b31ae9363 (doi: 10.6084/m9.figshare.11627190).

\section{Computed Tomography of Frozen Intact Specimens}

In addition to the osteological specimens examined at museum sites, the study obtained four recently deceased macropod specimens for medical CT scanning and histological examination. These included one Macropus rufus and three Macropus rufogriseus specimens which had died of natural causes at ZSL Whipsnade and London Zoos and had been stored frozen $\left(-20^{\circ} \mathrm{C}\right)$. We opportunistically examined these specimens in detail to test for variation within macropod patellae. The hindlimbs of each specimen were scanned using CT prior to dissection. Details of the scans are in Table S3 of Supplementary Text S1. Raw scan data are available at https://figshare.com/s/c3f5c083910b31ae9363 (doi: 10.6084/m9.figshare.11627190). 


\section{Dissection and Light microscopy of Frozen Specimens}

180

181

182

183

184

185

186

187

188

189

190

191

192

193

194

195

196

197

198

199

200

201

202

203

204

205

206

207

208

209

210

211

212

213

214

215

216

217

218

219

220

221

222

The right and left patellar tendons were harvested from two of the intact Macropus rufogriseus (specimens $1 \& 2$ ). The tendons dissected from the thawed cadavers were fixed in $10 \%$ neutral buffered formalin. Samples containing bone (diagnosed via imaging, above) were decalcified in $10 \%$ formic acid solution. All specimens were sectioned in the sagittal plane along the midline and directly lateral to the midline. The tendon sections were dehydrated and embedded in paraffin wax blocks. Microtome sections were cut between 4 and $6 \mu \mathrm{m}$. Sections were stained with Haematoxylin and Eosin, and Masson's trichrome. In the case of tendons where ossified patellar tissue was found to be present, sections were also stained with Safranin O/Fast Green for the identification of cartilage, and Von Kossa to highlight the presence of calcium salts. The histological sections were examined by light microscopy and images obtained via scanning at high resolution using a Leica SCN400F scanner.

\section{Evolutionary Reconstructions of Ancestral State}

Standard phylogenetic character mapping methods were used to reconstruct the evolutionary polarity of the patella in marsupials. A patellar character score was assigned to each species for which data had been obtained on the basis of the findings gathered from the literature, direct observations, radiographic/CT imaging and light microscopy; totalling 94 species. The patella was coded as absent $($ score $=0)$, fibrocartilage patelloid ( score $=1)$ or ossified $($ score $=2)$ for each species; and one additional "crown Eutheria" outgroup was scored as state 2; all following Samuels et al. (2017). In species where more than one state had been observed, or conflicting reports in the literature were unresolved, both possible states were included. For example, Macropus rufogriseus was coded as $1 / 2$ because both patelloid and ossified patella states were observed (see Results). A composite phylogenetic tree (here referred to as our "original tree") containing the species for which patellar data were available was obtained from the Timetree database, based on current literature (http://timetree.org; Hedges et al., 2006; see also MayCollado et al., 2015), with fossil outgroups added from Forasieppi (2009) and Bi et al. (2014, 2018). The data matrix is in Supplementary Data S1. We also conducted a sensitivity analysis of the assumed phylogeny by modifiying the fossil outgroups to match those adopted in Samuels et al. (2017).

The evolutionary history of the patella was reconstructed over the phylogenetic tree using the maximum likelihood (Mk1) model in Mesquite software (Maddison \& Maddison, 2017), with branch length calibrations following Samuels et al. (2017); unavailable branch length/divergence time data for extant taxa used the assumption that branch lengths were onehalf those connected to the next most deeply nested node. For comparison, a second reconstruction was generated using the maximum parsimony algorithm in Mesquite, with unordered character states; although we also checked this analysis by changing the reconstruction method to ordered character states (i.e. preferring stepwise changes from state 0 to 1 to 2 or the converse; not 0 to/from 2). We also re-ran analyses switching state " 0 " (no patella) coded fossil taxa to state " $0 / 1$ " (patelloid possible) to check sensitivity to the vagaries of preservation and interpretation of missing fibrocartilages. 


\section{Results}

224 A comprehensive literature review was the starting point for this study (Supplementary Text S1:

225 Table S7). To summarise: those higher-level clades for which there was evidence supporting the 226 presence of an ossified patella (state 2) included Caenolestidae, Notoryctidae, Thylacomyidae 227 and Peramelidae, with the possibility of an ossified patella in Tarsipedidae (Waterhouse, 1846; 228 Osgood, 1921) and the quokka Setonix brachyurus (Dawson et al., 1989; scored as state 1 or 2

229

230

231

232

233

234

235

236

237

238

239

240

241

242

243

244

245

246

247

248

249

250

251

252

253

254

255

256

257

258

259

260

261

262

263

264

265

266

267

268 due to ambiguity in specimens available). Even in the well-studied taxon Didelphis virginiana we found much confusion in the literature regarding patellar state, forcing us to code it ambiguously as state 1 or 2 . In a small number of species, including Vombatidae and Phascolarctidae, the patella was noted as absent (state 0 ) in some studies and in the form of a patelloid (state 1) in others. For example, in wombats, published descriptions ranged from absence of any sesamoid (Waterhouse, 1846; Lee \& Carrick, 1989) to an equivocal 0/1 character state (Home, 1808; Vogelnest and Allan, 2015) to presence of a patelloid (Macalister, 1870). The remainder of species for which data existed were scored as having a patelloid.

The most critical areas where data were sparse include the many South American opossums (excluding the genus Didelphis; only $\sim 7 \%$ of diversity), Microbiotheriidae (uncertain coding; see below), some species of Dasyuromorphia (e.g. only $\sim 10 \%$ of Dasyuridae), and the small Australian opossums and gliders (Acrobatidae, Tarsipedidae, Petauridae, Phalangeridae and Pseudocheiridae; uncertain coding for the former two and $\sim 6-25 \%$ diversity scored for the latter three). Overall, our final data matrix (see https://figshare.com/s/c3f5c083910b31ae9363 (doi: 10.6084/m9.figshare.11627190)) coded for about 72 species of marsupials, $\sim 19 \%$ of total diversity (Figure 2; Burgin et al., 2018; Mammal Diversity Database, 2020). Nonetheless, we sampled at least one species each from all family-ranked clades of extant Marsupialia; even about one-third of the relatively species-rich Macropodidae.

\section{Observations and Imaging of Osteological Specimens}

Firsthand observational data from preserved osteological specimens are in Table S4 of Supplementary Text S1. The nature of the patella was recorded for each specimen observed at the four museum collections visited. Details of specimens where CT or radiography was used for confirmation of patella mineralisation status are in Tables S5 and S6 of Supplementary Text S1. Examples of photographs and scans obtained are in Figures 3-6, with examples of radiographs in Figure 7.

Most findings from specimen-based examinations supported those from the literature review. Although it was not possible to conclusively confirm the presence of fibrocartilage without histological samples, the visual and imaging observations supported the presence of a fibrocartilage patelloid within the QF tendon for the majority of both Australian and American marsupials (Figures 3, 4). CT scans confirmed our visual observations for numerous marsupials (e.g. Figure 4), demonstrating relatively low density and homeogeneous structure of the QF tendon and patelloid (Figures 3,5) vs. the ossified patella or other bones (Figure 6). Our analysis supported the literature in finding likely patelloids in the dessicated QF tendons of both hindlimbs of a well-preserved specimen of the now-extinct Thylacinus cynocephalus (Figure 5). The form of the patella of this species was briefly mentioned as a patelloid by Cunningham (1882) and Wood (1924), which Warburton et al. (2019) supported based on a more exhaustive study (also character coding in Forasieppi (2009)). We thus accepted these character state 
269

270

271

272

273

274

275

276

277

278

279

280

281

282

283

284

285

286

287

288

289

290

291

292

293

294

295

296

297

298

299

300

301

302

303

304

305

306

307

308

309

310

311

312

313

codings where available evidence best supported interpretation as fibrocartilage patelloid vs. alternatives (also see Methods). Importantly, observations were made in numerous species for which there had previously been no data recorded. Unfortunately, there was still a large number of species for which we observed no specimens or found no clear literature descriptions; our sampling of Marsupialia was necessarily incomplete (see sampling summary above).

Ossified patellae were evident in Caenolestidae, Notoryctidae, Thylacomyidae and Peramelidae (Figure 6), which also supported the literature review. The structure of the patella in these taxa is similar and also similar to that in other mammals (Samuels et al., 2017), consistent with common developmental mechanisms (e.g. endochondral ossification). We have provided among the first detailed images (external and internal morphology) of the ossified patella in these taxa, in addition to the patelloids in others.

\section{Computed Tomography and Visual Observations of Frozen Specimens}

CT scans of the wet Macropus rufogriseus and Macropus rufus specimens revealed the presence of an ossified patella in the left hindlimb of one Macropus rufogriseus (specimen 1; Figure 8). The basic structure of this patella that was evident in CT images (e.g. cortical shell, trabecular interior) matched the structure evident from CT scans of other marsupials with ossified patellae, or the structure of the lateral sesamoid (Figures 3,6,8). Upon subsequent dissection, this ossified patella was clearly evident (Figure 8). In the remaining three specimens of Macropus, a patelloid was observed on the CT scans and subsequently confirmed to be fibrocartilage in one specimen (specimen 2) by dissection and histological examination (Figure 9).

\section{Light Microscopy of Frozen Specimens}

Histological examination confirmed the presence of an ossified patella in the left QF tendon of specimen 1 of Macropus rufogriseus (Figures 10,11). The majority of the patella was composed of cancellous bone, with cortical bone comprising the superficial third (Figure 11A); approximately typical for an ossified mammalian patella. The Safranin O/Fast Green staining highlighted a layer of articular hyaline cartilage covering the deep surface: the surface in articulation with the femur. Staining with Von Kossa identified the presence of calcium salts, with a central region of increased calcium density. In contrast, the right QF tendon of specimen 1, and both QF tendons of specimen 2, contained a typical fibrocartilage patelloid (Figure 10). The presence of cartilage was best highlighted by staining with Safranin O/Fast Green, while collagen fibres were most clearly illustrated with Masson's trichrome. As expected, the majority of the patelloid stained poorly with Von Kossa, illustrating that the tissue was not ossified (Figure 11B). However, stain accumulated in one small region in the centre of the tendon, suggesting a small amount of nascent mineralisation/ossification there.

\section{Evolutionary Reconstructions}

Figure 12 illustrates the reconstructed evolution of the patella in marsupials, according to the maximum likelihood (Mk1) and parsimony algorithms. Under the maximum likelihood model, absence of any patellar sesamoid (mineralised or patelloid) was reconstructed as the most likely ancestral state for Metatheria. Sparassodonts, as in Samuels et al. (2017), were united by an apparently independently evolved ossification of the patella (e.g. Sinclair, 1905, Wood, 1924; Argot, 2004; Forasieppi, 2009). Our reconstruction indicates that the common ancestor at the 
314 root of the crown group marsupial tree was most likely (62\%; greater at deeper nodes 3-6 in

315 Figure 12) to have evolved a fibrocartilage patelloid (i.e. character state 1), which has been 316 maintained in the majority of sampled marsupial species. The likelihood of an ancestral patelloid 317 state in marsupials increased (to 97\%; same if using the Samuels et al. (2017) tree) if a patelloid 318 state was considered possible in all non-marsupial metatherians lacking ossified patellae (e.g. 319 Herpetotherium coded 0/1 rather than 0 ). We found three separate instances of evolution of an 320 ossified patella (i.e. character state 2) from the ancestral patelloid within the crown group,

321

322

323

324

325

326

327

328

329

330

331

332

333

334

335

336

337

338

339

340

341

342

343

344

345

346

347

348

349

350

351

352

353

354

355

356

357

358

359 occurring at the nodes for Caenolestidae, Notoryctidae and Peramelemorphia, according to the maximum likelihood reconstruction (with our original tree and that of Samuels et al. (2017)). Hence an ossified patella may have evolved at least four times in major clades within Metatheria.

There was also one apparent instance of loss of a patelloid (i.e. reversal to character state 0), in Dromiciops (Microbiotheriidae), however this was only suggested by observations from a single study (Szalay \& Sargis, 2001). Similarly, for the closely-related Vombatidae and Phascolarctidae, and for Sarcophilus harrisii and Metachirus nudicaudatus, some sources noted complete absence of any patella or patelloid within the quadriceps tendon (full details in Supplementary Text S1). This evidence leads to the tentative speculation that a reversal from patelloid to absence of any sesamoid occurred more than three times in our sample of Marsupialia, although our coding for this character state was left equivocal (state 0 or 1; Figure 12), as some more recent sources imply that a patelloid may indeed be present. Indeed, dissections of two male and two female adult koalas (Phascolarctos cinereus) indicated the bilateral presence of patelloids, not ossified patellae (Hazel Richards, Monash University, pers. comm. 2019; confirmed by CT scans), as shown in Figure 13. There were several instances of ossified patellae being observed in (individuals of) a single species, despite other species within the same family-rank clade possessing a fibrocartilage patelloid. These included Didelphis virginiana, Tarsipes rostratus and multiple macropod species (e.g. Setonix brachyurus). Likewise, here, we coded these states as equivocal until stronger sampling can be conducted. Regardless, transformations from an unossified patelloid to patella appeared common in our maximum likelihood analyses for Marsupialia.

In comparison with our maximum likelihood analyses, maximum parsimony analysis with unordered character states (Figure 12; branch colours) presented a very different evolutionary pattern. An ossified patella united most of Metatheria [Sparassodonta + Marsupialia] as an ancestral state. Next, there were three independent reductions to a patelloid (i.e. no ancestral transformations from patelloid to patella for major clades) along the lineages to Didelphidae, Dasyuridae/Dasyuromorphia and Diprotodontia. Otherwise the results were similar. Forcing the character states to evolve in an ordered regime produced results more concordant with the maximum likelihood analysis: ancestral fibrocartilage for [Sparassodonta + Marsupialia], then two independent origins of ossified patellae in sparassodonts and caenolestids, although it was ambiguous whether ossified patellae were homologous or homoplastic for [Dasyuridae + Peramelemorphia + Notoryctidae] on the tree used, involving one or two more origins of an ossified patella, totaling 3-4 occurrences. Using a phylogeny concordant with that of Samuels et al. (2017) produced identical results for both parsimony models (unordered/ordered).

When we coded all fossil taxa originally scored as having "patella absent" (= state 0$)$ to "patella absent"/"patelloid present" (= state 0/1) under maximum parsimony, we obtained some similar

Peer] reviewing PDF | (2020:01:44905:1:1:NEW 21 Jul 2020) 
360

361

362

363

364

365

366

367

368

369

370

371

372

373

374

375

376

377

378

379

380

381

382

383

384

385

386

387

388

389

390

391

392

393

394

395

396

397

398

399

400

401

402

403

404

405

results to those before this change of assumptions. Unordered parsimony produced an ambiguous state 1/2 (patelloid/ossified patella present); not 2; at the node [Sparassodonta + Marsupialia], then equivocal states for some of the more inclusive branches within Marsupialia, so 2-4 total independent ossification events could have occurred. Ordered parsimony produced the same 3-4 origins of an ossified patella as above. Conducting this same procedure on the Samuels et al. (2017) tree, again we found ambiguity under unordered parsimony, with state $1 / 2$ prevailing up to [Sparassodonta + Marsupialia], then an uncertain state $1 / 2$ across major nodes until Diprotodontia (state 1); leaving it uncertain whether an ossified patella evolved independently or not in caenolestids, Notoryctidae, or Peramelemorphia (Figure S1). Yet again, ordered parsimony gave results concordant with others using that assumption.

Overall, then, depending on which tree and evolutionary reconstruction method was adopted, we tended to obtain 3-4 origins of an ossified patella in Sparassodonta + Marsupialia. Nonetheless, it remained feasible that an ossified patella might have reversed to fibrocartilage 3 times (with unordered parsimony and our original tree and character scores).

\section{Discussion}

We conducted an in-depth analysis of the form of the marsupial patella across Marsupialia, using observations from both osteological and wet specimens, combined with critical analysis of the available literature (Table S7 of Supplementary Text S1). Our finer-level dataset did not clearly support the hypothesis of an ancestrally-ossified patella in marsupials, proposed by a previous, less detailed study also using maximal likelihood character mapping methods (Samuels et al., 2017). The new maximum likelihood ancestral state reconstruction indicated evolution of an ossified patella from a fibrocartilage patelloid at three separate instances during marsupial evolution. However, our parsimony-based analysis contradicted this reconstruction in that an ossified patella evolved first in Metatheria, and then was independently transformed into a patelloid at least three times; altered if we assumed parsimony with ordered character polarity. Our conclusions thus hinge on which evolutionary algorithm is preferred. Regardless, our findings concur that there is extensive homoplasy for the ossified patellar sesamoid in Metatheria.

We have also discovered the presence of an ossified patella in one individual of Macropus rufogriseus, a species previously stated to possess a fibrocartilage patelloid(Reese et al., 2001). Intriguingly, this M. rufogriseus individual exhibited two different patellar states: the right knee had a fibrocartilage patelloid, as anticipated from the literature, whilst the left had a very well-differentiated bony patella complete with articular hyaline cartilage. The discrepancy within these species, and particularly within an individual, may go some way towards explaining apparent contradictions in literature data. This may, in part, be due to the vague references found in certain older studies. However, it also raises the possibility that several marsupial species, previously thought to possess a patelloid, are actually able to develop an ossified patella, perhaps in different environments (e.g. mechanical, developmental) or simply due to random variation. The fact that different patellar states exist among closely related species, or even within individuals, highlights the complexity of marsupial patellar evolution. Perhaps developmental potential for an ossified patella may exist in most or all marsupial species, with ossification only actually occurring in certain cases. 
406

The results presented above challenge the frequently quoted statement that "all marsupials, except bandicoots and the bilby" lack an ossified patella (Reese et al., 2001; Inamassu et al., 2017; Vogelnest \& Allan, 2015). The very clear presence of a bony patella in the Caenolestidae and Notoryctidae (e.g. Thompson \& Hillier, 1905; Figure 6) is sufficient evidence to the contrary. Furthermore, the observation of an ossified patella in a single Macropus individual, combined with some ambiguity in literature noted above and in the Supplementary Text S1 (Table S7), cautions against the use of broad cross-species generalisations which implicitly assume $100 \%$ evolutionary fixation of character states within/between species. Sarin et al. (1999) examined the incidence of two sesamoids (the fabella and os peroneum) in primates, finding intra-species variation in the occurrence of the former sesamoid (vs. quasi-constancy of the latter), and inferring a decline in its incidence along the primate stem lineage and then secondary increase in Homo. Berthaume et al. (2019) then found remarkable increases in the ossification of the fabella over the past 150 years in human populations, attributing this to improved health and nutrition favouring larger body sizes and thus increased mechanical stimuli.

Our results indicate a complex pattern of evolution and development of this sesamoid, with patellar states apparently not constant in all clades, and with certain individuals capable of developing an ossified patella in species otherwise typically possessing a patelloid. Thus, there is polymorphism in several marsupial taxa, as hinted at by Samuels et al. (2017), although the biological mechanisms underlying this variation remain uncertain - and certainly deserving deeper mechanistic studies. A genetic basis is hypothesised for many sesamoids, with further development driven by epigenetic factors (see Abdala et al., 2019 for recent review). Repeated evolutionary losses and regains of an ossified patella are thus very plausible for marsupials, perhaps through evolutionary 'maintenance' of a transitional structure like the patelloid. Marsupials are known to have apomorphically delayed hindlimb development (Hamrick, 1999; Sánchez-Villagra, 2002; Garland et al., 2007; Sears, 2009) compared with other mammals, so we speculate that this heterochronic shift might have also impacted patellar development and its evolution. New data on patellar development in marsupials are urgently needed, especially in light of novel recent insights into the tissue origins and molecular controls of patellar development in mammals (Eyal et al., 2015,2019; Márquez-Flórez et al., 2018; Samuels \& Campeau, 2019).

The maximum likelihood reconstruction of Samuels et al. (2017) suggested a single origin of a bony patella in Metatheria, prior to the divergence of Marsupialia and Sparassodonta, followed by reduction to a fibrocartilage patelloid in most marsupials (e.g. ancestral Diprotodontia) and re-ossification in some deeper lineages (e.g. Tarsipedidae). This was found to be more likely than multiple instances of bony patellar evolution (Samuels et al., 2017). Our present study focused on data at the species level and much more expansive sampling of individual specimens (Supplementary Text S1; Tables S1-S7), rather than just family-level clades as in Samuels et al. (2017); and we placed Caenolestidae and Didelphidae as sister taxa in our phlogeny (Figs. 2, 9) rather than successive branches (as per our source of the phylogeny; see Methods). Alternative phylogenies (e.g. Bininda-Emonds et al. 2007; Meredith et al., 2011; O'Leary et al., 2013) would alter our results at least slightly, and resolution of this issue requires better consensus among mammalian phylogenies in general. We found conflicting evolutionary patterns that only concur with Samuels et al.'s (2017) if maximum parsimony (with unordered character states) rather than likelihood (or ordered character states) is adopted. In the latter case,

PeerJ reviewing PDF | (2020:01:44905:1:1:NEW 21 Jul 2020) 
452 maximum likelihood (and ordered-state parsimony) favoured the more conventional scenario that

453 Metatheria lacked an ossified patellar sesamoid ancestrally, but may have possessed a patelloid

454 (the origin of which is dependent on coding in extinct non-marsupial metatherians). Later, three

455 or more different marsupial clades independently ossified the patelloid that was ancestral for the

456 crown group. Resolving this discordance will depend upon what algorithm is favoured (we

457 suggest that the latter results are the most plausible), but also on acquiring more high-resolution

458 data across Metatheria.

459

460

461

We, however, contend that coding the patellar states as ordered is more plausible given available ontogenetic data and mechanobiological theory (e.g. Sarin \& Carter, 2000; MárquezFlórez et al., 2018; Abdala et al., 2019). Reciprocally, the general congruence between those results and maximum likelihood offer some reassurance that an evolutionary sequence from little/no patella to "patelloid" to 3+ parallel evolutions of bony patellae may be the most reasonable conclusion at present. Furthermore, maximum likelihood analyses tend to be favoured for studies of character evolution (Schluter et al., 1997; Pagel, 1999; Oakley, 2003). Nonetheless, this complex question of patellar evolution in metatherians, which has long been plagued by a seeming bias toward simple answers, deserves continued and deeper inquiry. There are interesting broader stakes for evolutionary developmental biology and biomechanics. For example, marsupial patellae are relevant to the concept of "traction epiphyses", that 'new' bones may originate by disconnection from or be 'lost' by fusion to long bones (see reviews in Vickaryous and Olsen, 2007; Abdala et al., 2019). Whether ossified or fibrocartilaginous, the patella of marsupials and other mammals might have originated as a traction epiphysis from the femur (Eyal et al., 2015, 2017) or the tibia. In cases where the patella was entirely lost, it might have even merged with one of those bones. Sufficient developmental data do not, to our knowledge, exist to enable testing of this idea at present.

Unfortunately, several species were unavailable for inclusion in this study, and occasionally only single specimens of a particular species were observed. Further data on the form of the patella in marsupials and their metatherian cousins are still required, in order to further clarify the pattern of patellar evolution in marsupials. Additionally, the age (or even gross ontogenetic stage; other than near-adult) of the preserved specimens studied was largely unknown. Previously, Szalay \& Sargis (2001) had suggested that ossification may occur in older individuals of some species, referring specifically to Didelphis virginiana. However, examination of an ontogenetic series of specimens, perhaps of multiple species, would be required to resolve this matter. Such an age-related correlation is supported by the prevalence of fabellar sesamoid ossification in humans (Berthaume and Bull, 2020), for example.

The (tentative) conclusion that a bony patella evolved, or was reacquired, at multiple times in divergent marsupial species raises interesting questions regarding the function of this sesamoid. Previous studies examining the form of the marsupial patella have varied in their conclusions about how the presence of a patella or patelloid relates to the ecology and behaviour of the species studied. Holladay et al. (1990) attributed the presence of a patelloid in macropods to their pattern of locomotion. In contrast, Reese et al. (2001: p.293) challenged that "the lack of a bony patella is typical for marsupials", but the different patelloid "types" they observed result from the different mechanical stresses acting on the knee joint of different species. Abdala et al. (2017) suggested that the presence of a fibrocartilaginous patella in some frog species might be 
498

499

500

501

502

503

504

505

506

507

508

509

510

511

512

513

514

515

516

517

518

519

520

521

522

523

524

525

526

527

528

529

530

531

532

533

534

535

536

537

538

539

540

correlated with jumping biomechanics, mirroring some speculations from studies of the fibrocartilaginous patelloid in marsupials (Holladay et al., 1990; Reese et al., 2001). However, to the degree that a fibrocartilaginous patelloid is present in marsupials, it is a plesiomorphic trait for lineages that hop, rather than co-evolving with hopping gaits; hence we doubt this correlation for marsupials. We did not carry out functional analysis of the marsupial patella here; however, the observations made here certainly warrant future functional studies. In particular, the question of why would an ossified patella form in some individuals of a species, while others possess a fibrocartilage patelloid, remains unresolved and would require quantitative biomechanical analyses to test. This is an exciting foundation for future potential studies in evolutionary developmental biomechanics.

\section{Conclusions}

New, finer-scale evolutionary reconstructions presented here suggest that an unossified patelloid was ancestral for marsupials, with repeated gains (three or more) of an ossified patella within crown group Marsupialia. Our novel observation of an ossified patellae in an individual of Macropus rufogriseus challenges the typical generalisation regarding the fibrocartilage nature of the marsupial patelloid. However, a large number of species remain unstudied, including several small species of possum, for which preservation of the patella is difficult to ascertain or ensure in museum specimens. Furthermore, the mechanistic basis of of patellar sesamoid "evo-devo" and biomechanics remains an important open question for explaining how and why patellar forms changed repeatedly across metatherian evolution.

\section{Acknowledgements}

We thank ZSL London Zoo and Whipsnade Zoo for donation of deceased macropod specimens, in particular Dr. Edmund Flach for preparing specimens for collection. Anjali Goswami gave helpful support to this project in early stages. Kind thanks to Roberto Portela Miguez for carrying out the specimen radiography and other important assistance at NHMUK, and to Ket Smithson, Rob Asher and Matt Lowe for specimen micro-CT scanning and access at UMZC. Hazel Richards's provision of images and details on koala dissections is greatly appreciated. We appreciate the constructive reviews from Michael Berthaume, Nicolás Reyes-Amaya and an anonymous reviewer.

\section{References}

Abdala V, Vera MC, Amador LI, Fontanarrosa G, Fratani J, Ponssa ML. 2019. Sesamoids in tetrapods: the origin of new skeletal morphologies. Biological Reviews, published online. doi: 10.1111/brv.12546

Abdala V, Vera MC, Ponssa ML. 2017. On the presence of the patella in frogs. Anatomical Record 300: 1747-1755.

Peer) reviewing PDF | (2020:01:44905:1:1:NEW 21 Jul 2020) 
541 Alexander RM, Dimery NJ. 1985. The significance of sesamoids and retro-articular processes for

542

543

544

545

546

547

548

549

550

551

552

553

554

555

556

557

558

559

560

561

562

563

564

565

566

567

568

569

570

571

572

573

574

575

576

577

578

579

580

581

582

583

584

585

586

the mechanics of joints. Journal of Zoology 205(3): 357-371.

Aplin KP, Archer M. 1987. Recent advances in marsupial systematics with a new syncretic classification. In: Archer M, ed. Possums and Opossums: Studies in Evolution. Sydney: Surrey Beatty and the Royal Zoological Society of New South Wales, 15-72.

Argot C. 2004. Functional-adaptive analysis of the postcranial skeleton of a Laventan borhyaenoid, Lycopsis longirostris (Marsupialia, Mammalia). Journal of Vertebrate Paleontology 24(3): 689-708.

Berthaume MA, Bull AM. 2020. Human biological variation in sesamoid bone prevalence: the curious case of the fabella. Journal of Anatomy 236(2): 228-242.

Berthaume MA, Di Federico E, Bull AMJ. 2019. Fabella prevalence rate increases over 150 years, and rates of other sesamoid bones remain constant: a systematic review. Journal of Anatomy 235: 67-79. doi: 10.1111/joa. 12994

Bi S, Wang Y, Guan J, Sheng X, Meng J. 2014. Three new Jurassic euharamiyidan species reinforce early divergence of mammals. Nature 514(7524): 579-584.

Bi S, Zheng X, Wang X, Cignetti NE, Yang S, Wible JR. 2018. An Early Cretaceous eutherian and the placental-marsupial dichotomy. Nature 558(7710): 390-395.

Bininda-Emonds OR, Cardillo M, Jones KE, MacPhee RD, Beck RM, Grenyer R, Price SA, Vos RA, Gittleman JL, Purvis, A. 2007. The delayed rise of present-day mammals. Nature 446(7135): 507.

Burgin CJ. Colella JP, Kahn PL, Upham NS. 2018. How many species of mammals are there?. Journal of Mammalogy, 99: 1-14.

Cunningham DJ. 1882. Report on some points in the anatomy of the thylacine (Thylacinus cynocephalus), cuscus (Phalangista maculata), and phascogale (Phascogale calura), collected during the voyage of HMS Challenger in the years 1873-1876: With an account of the comparative anatomy of the intrinsic muscles and the nerves of the mammalian pes. Longmans.

Dawson TJ, Finch E, Freedman L, Hume ID, Renfree MB, Temple-Smith PD. 1989. Morphology and physiology of the Metatheria. In: Walton DW, Richardson BJ. Fauna of Australia. Volume 1B: Mammalia. Australian Government Publishing Service.

Eyal S, Blitz E, Shwartz Y, Akiyama H, Ronen S, Zelzer E. 2015. On the development of the patella. Development 142(10): 1831-1839.

Eyal S, Rubin S, Krief S, Levin L, Zelzer E. 2019. Common cellular origin and diverging developmental programs for different sesamoid bones. Development 146(4): dev167452.

PeerJ reviewing PDF | (2020:01:44905:1:1:NEW 21 Jul 2020) 
587 Fatima M, Scholes CJ, Zhong E, Kohan L. 2019. Towards a dynamic model of the kangaroo

588 knee for clinical insights into human knee pathology and rreatment: Establishing a static

589 biomechanical profile. Biomimetics 4(3):52. doi: 10.3390/biomimetics 4030052

590

591

592

593

594

595

596

597

598

599

600

601

602

603

604

605

606

607

608

609

610

611

612

613

614

615

616

617

618

619

620

621

622

623

624

625

626

627

628

629

630

631

Flores DA. 2009. Phylogenetic analyses of postcranial skeletal morphology in didelphid marsupials. Bulletin of the American Museum of Natural History 320: 1-81.

Forasiepi AM. 2009. Osteology of Arctodictis sinclairi (Mammalia, Metatheria, Sparassodonta) and phylogeny of Cenozoic metatherian carnivores from South America. Monografías del Museo Argentino de Ciencias Naturales 6: 1-174.

Garland K, Marcy A, Sherratt E, Weisbecker V. 2017. Out on a limb: bandicoot limb co-variation suggests complex impacts of development and adaptation on marsupial forelimb evolution. Evolution \& Development 19(2): 69-84.

Hamrick MW. 1999. Development of epiphyseal structure and function in Didelphis virginiana (Marsupiala, Didelphidae). Journal of Morphology 239(3): 283-296.

Haxton H. 1944. The patellar index in mammals. Journal of Anatomy 78: 106-107.

Hedges SB, Dudley J, Kumar S. 2006. Timetree: a public knowledge-base of divergence times among organisms. Bioinformatics 22(23): 2971-2972.

Holladay SD, Smith BJ, Smallwood JE, Hudson LC. 1990. Absence of an osseous patella and other observations in Macropodidae stifle. Anatomical Record 226(1): 112-114.

Home E. 1808. An account of some peculiarities in the anatomical structure of the Wombat, with observations on the female organs of generation. Philosophical Transactions of the Royal Society of London 98: 304-312.

Inamassu LR, Mamprim MJ, Dadalto CR, Cavaletti FC, Mello MC, Schimming BC. 2017. Absence of bony patella in the white-eared opossum (Didelphis albiventris): Morphology and diagnostic imaging. Anatomia, Histologia, Embryologia 46(6): 611-614.

Johnson KA, Walton DW. 1989. Notoryctidae. In: Walton DW, Richardson BJ. Fauna of Australia. Volume 1B: Mammalia. Australian Government Publishing Service.

Lee AK, Carrick FN. 1989. Phascolarctidae. In: Walton DW, Richardson BJ. Fauna of Australia. Volume 1B: Mammalia. Australian Government Publishing Service.

Macalister A. 1870. XVI.--On the myology of the Wombat (Phascolomys wombata) and the Tasmanian Devil (Sarcophilus ursinus). Annals and Magazine of Natural History 4: 153-173.

Maddison WP, Maddison DR. 2017. Mesquite: a modular system for evolutionary analysis. Available at http://www.mesquiteproject.org.

632 
633 Mammal Diversity Database. 2020. www.mammaldiversity.org. American Society of

634 Mammalogists. Accessed 2 March 2020.

635

636

637

638

639

640

641

642

643

Márquez-Flórez K, Shefelbine S, Ramírez-Martínez A, Garzón-Alvarado D. 2018.

Computational model for the patella onset. PloS one 13(12): e0207770.

May-Collado LJ, Kilpatrick CW, Agnarsson I. 2015. Mammals from 'down under': a multi-gene species-level phylogeny of marsupial mammals (Mammalia, Metatheria). PeerJ 3: e805.

Meredith RW, Janečka JE, Gatesy J, Ryder OA, Fisher, CA, Teeling EC, Goodbla A, Eizirik E, 644 TL, Robinson TJ, Buurk-Herick A, Westerman M, Ayoub NA, Springer MS, Murphy WJ. 2011.

645

646

647

648

649

650

651

652

653

654

655

656

657

658

659

660

661

662

663

664

665

666

667

668

669

670

671

672

673

674

675

676

677 Impacts of the Cretaceous terrestrial revolution and KPg extinction on mammal diversification. Science 334(6055): 521-524.

Oakley, T. H. 2003. Maximum likelihood models of trait evolution. Comments on Theoretical Biology 8: 1-17.

O'Leary MA, Bloch JI, Flynn JJ, Gaudin TJ, Giallombardo A, Giannini NP, Goldberg SL, Kraatz BP, Luo ZX, Meng J, Ni X, Novacek MJ, Perini FA, Randall ZS, Rougier GW, Sargis EJ, Silcox MT, Simmons NB, Spaulding M, Velazco PM, Weksler M, Wible JR, Cirranello AL. 2013. The placental mammal ancestor and the post-K-Pg radiation of placentals. Science 339: 662-667.

Osgood WH. 1921. A monographic study of the American Marsupial, Caenolestes. Chicago: Field Museum of Natural History. Zoological Series 14(1):1-162.

Pagel M. 1999. Inferring the historical patterns of biological evolution. Nature 401(6756): 877884.

Reese S, Pfuderer UR, Bragulla H, Loeffler K, Budras K-D. 2001. Topography, structure and function of the patella and the patelloid in marsupials. Anatomia, Histologia, Embryologia 30(5): 289-294.

Samuels ME, Regnault S, Hutchinson JR. 2017. Evolution of the patellar sesamoid bone in mammals. PeerJ, 5, e3103.

Samuels ME, Campeau PM. 2019. Genetics of the patella. European Journal of Human Genetics 27: $671-680$.

Sánchez-Villagra MR. 2002. Comparative patterns of postcranial ontogeny in therian mammals: an analysis of relative timing of ossification events. Journal of Experimental Zoology 294(3): 264-273.

678

Sarin VK, Carter DR. 2000. Mechanobiology and joint conformity regulate endochondral ossification of sesamoids. Journal of Orthopaedic Research 18(5): 706-712. 
679 Sarin VK, Erickson GM, Giori NJ, Bergman AG, Carter DR. 1999. Coincident development of 680 sesamoid bones and clues to their evolution. Anatomical Record 257(5): 174-180.

681

682

683

684

685

686

687

688

689

690

691

692

693

694

695

696

697

698

699

700

701

702

703

704

705

706

707

708

709

710

711

712

713

714

715

716

717

718

719

720

721

722

723

Schluter D, Price T, Mooers AØ, Ludwig D. 1997. Likelihood of ancestor states in adaptive radiation. Evolution 51(6): 1699-1711.

Sears KE. 2009. Differences in the timing of prechondrogenic limb development in mammals: the marsupial-placental dichotomy resolved. Evolution 63(8): 2193-2200.

Sinclair WJ. 1905. The marsupial fauna of the Santa Cruz beds. Proceedings of the American Philosophical Society 44(179): 73-81.

Sonntag CF. 1922. On the myology and classification of the wombat, koala, and phalangers. Proceedings of the Zoological Society of London 92: 863-866.

Szalay FS, Sargis EJ. 2001. Model-based analysis of postcranial osteology of marsupials from the Palaeocene of Itaborai (Brazil) and the phylogenetics and biogeography of Metatheria.

Geodiversitas 23: 139-302.

Thompson P, Hillier WT. 1905. Myology of the hindlimb of the marsupial mole. Journal of Anatomy and Physiology 39(Pt 3): 308-331.

Vickaryous MK, Olson WM. 2007. Sesamoids and ossicles in the appendicular skeleton. In: Hall BK, ed. Fins into Limbs. Chicago: University of Chicago.

Vogelnest L, Allan G. 2015. Radiology of Australian Mammals. Csiro Publishing.

Walton DW, Richardson BJ. 1989. Fauna of Australia. Volume 1B: Mammalia. Australian Government Publishing Service.

Warburton NM. 2006. Functional morphology of marsupial moles (Marsupialia; Notoryctidae). Verhandlungen des Naturwissenschaftlichen Vereins 42: 39-149.

Warburton NM, Travouillon KJ, Camens AB. 2019. Skeletal atlas of the Thylacine (Thylacinus cynocephalus). Palaeontologia Electronica 22.2.29A: 1-56. https://doi.org/10.26879/947

Waterhouse GR. 1846. A Natural History of the Mammalia. Vol I. Containing the Order Marsupialia. Baillière.

Windle BC, Parsons FG. 1898. On the anatomy of Macropus rufus. Journal of Anatomy and Physiology 32(Pt 1): 119-134.

Wood HE. 1924. The position of the" Sparassodonts": with notes on the relationships and history of the Marsupialia. Bulletin of the American Museum of Natural History 51(4): 77-101.

Peer) reviewing PDF | (2020:01:44905:1:1:NEW 21 Jul 2020) 
Figure 1

Evolutionary divergence within Mammalia (e.g. May-Collado et al., 2015).

Approximate timings in MYA (million years ago). Divergence time estimates from Timetree database (http://www.timetree.org; Hedges et al., 2006).

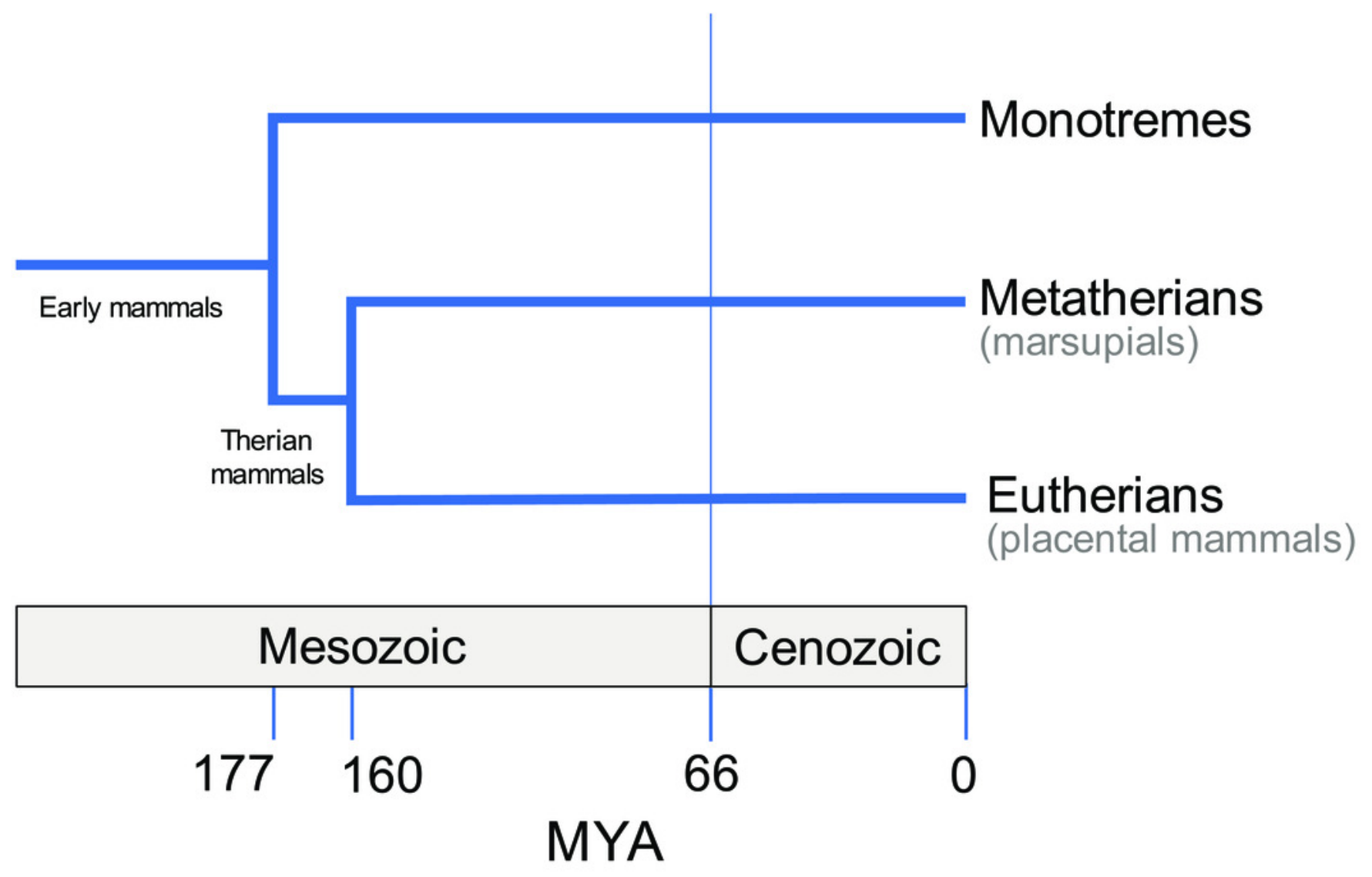


Figure 2

Summary cladogram of the main extant marsupial family-rank clades sampled in this study.

Evolutionary relationships are according to the Timetree database (http://www.timetree.org;

Hedges et al., 2006).

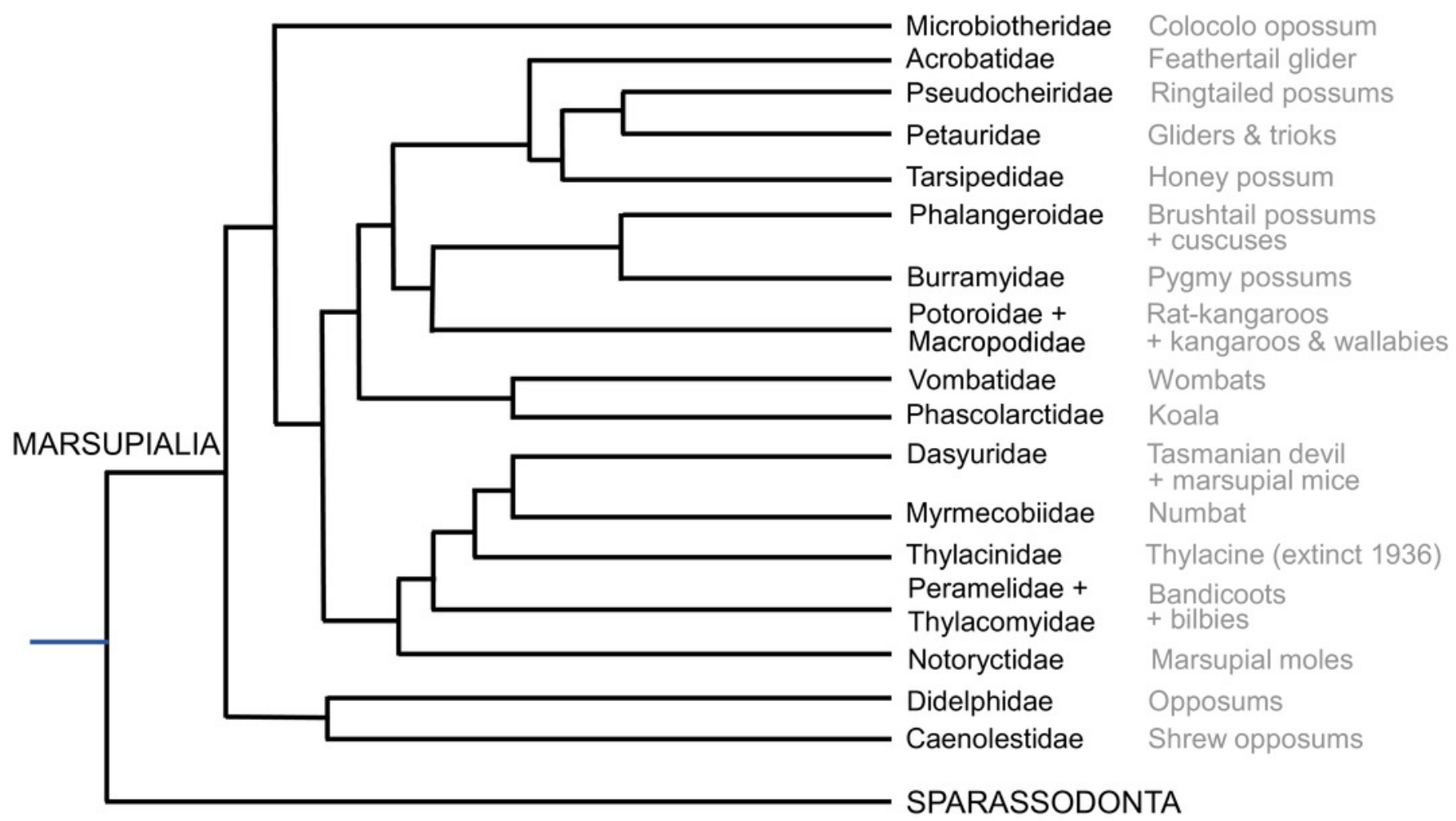




\section{Figure 3}

Example images of marsupial specimens with patelloids (Supplementary Text S1: Tables $\mathrm{S} 1, \mathrm{~S} 5)$.

(A, B, C): Thylogale billiardierii (UMZC specimen A12.50-3) pademelon -- no ossified patella as shown in photograph in $\mathrm{A}$ (scale bar squares $=1 \mathrm{~cm}$ ), 3D reconstruction from micro-CT scans of hindlimbs in B and longitudinal section from micro-CT scans with grey (fibro)cartilage density corresponding to patelloid in C. (D,E): Petaurus breviceps (UMZC specimen A9.40-2) sugar glider. (D) Skeleton in right side view showing left and right patelloids. (E) Micro-CT scan of hindlimbs in frontal plane view showing (on left) right hindlimb/knee joint and (on right) QF tendon/patelloid. Green arrows point to location of patelloid where visible. Note lower density of QF tendon and patelloid (greyer shades) vs. bony tissue (whiter shades) in C and E. Labels: "fem" = femur; "Is" = lateral sesamoid; "pes" = pes (hindfoot); "tf" = tibia and fibula. Red horizontal scale bars in $C$ and $E$ are $20 \mathrm{~mm}$ and $4 \mathrm{~mm}$. 

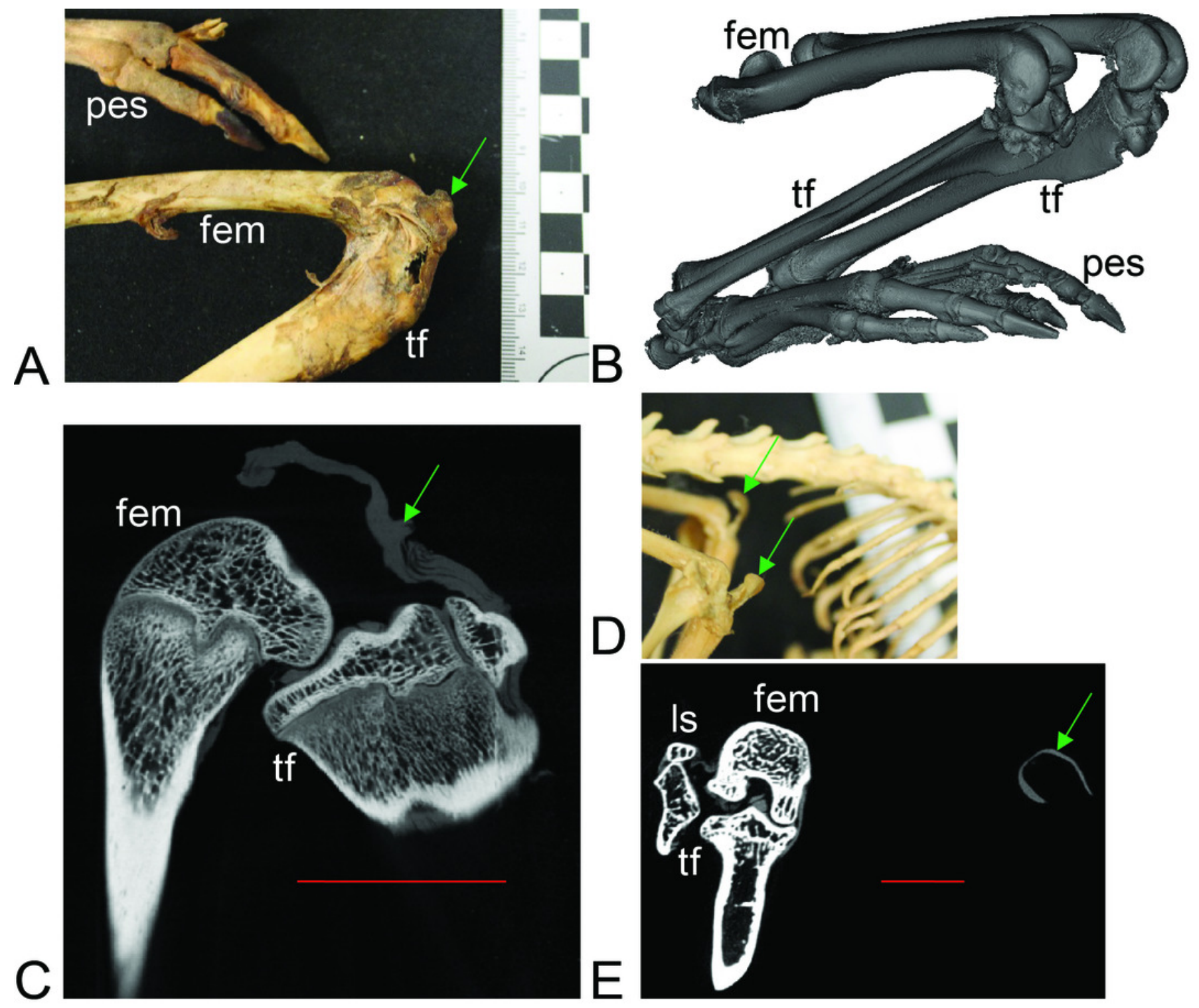


\section{Figure 4}

Other example images of marsupial specimens with patelloids (Supplementary Text S1: Table S4).

(A) Vombatus ursinus (NHMUK specimen 196.4.6.29.1) wombat, left hindlimb. (B) Trichosurus vulpecula (NHMUK specimen 89.266) brushtail possum, left hindlimb. (C) Bettongia lesueur (NHMUK specimen 277.70) boodie, right (top) and left (bottom) hindlimbs. (D) Macropus agilis (NHMUK specimen 70.368) agile wallaby, left and right hindlimbs. (E) Onychogale fraenata (UMZC specimen A12.59/2) bridled wallaby, right hindlimb. (F) Dasyurus viverrinus (UMZC specimen A6.11-6) quoll, right hindlimb. (G) Aepyprymnus rufescens (UMZC specimen A12.77-3) rufous bettong, left and right hindlimbs. Green arrows point to location of patelloid where visible. Scale bar squares $=1 \mathrm{~cm}$; note some photos are at oblique angles to scale and hence not precisely to-scale. Labels: "fem" = femur; "tf" = tibia and fibula. 

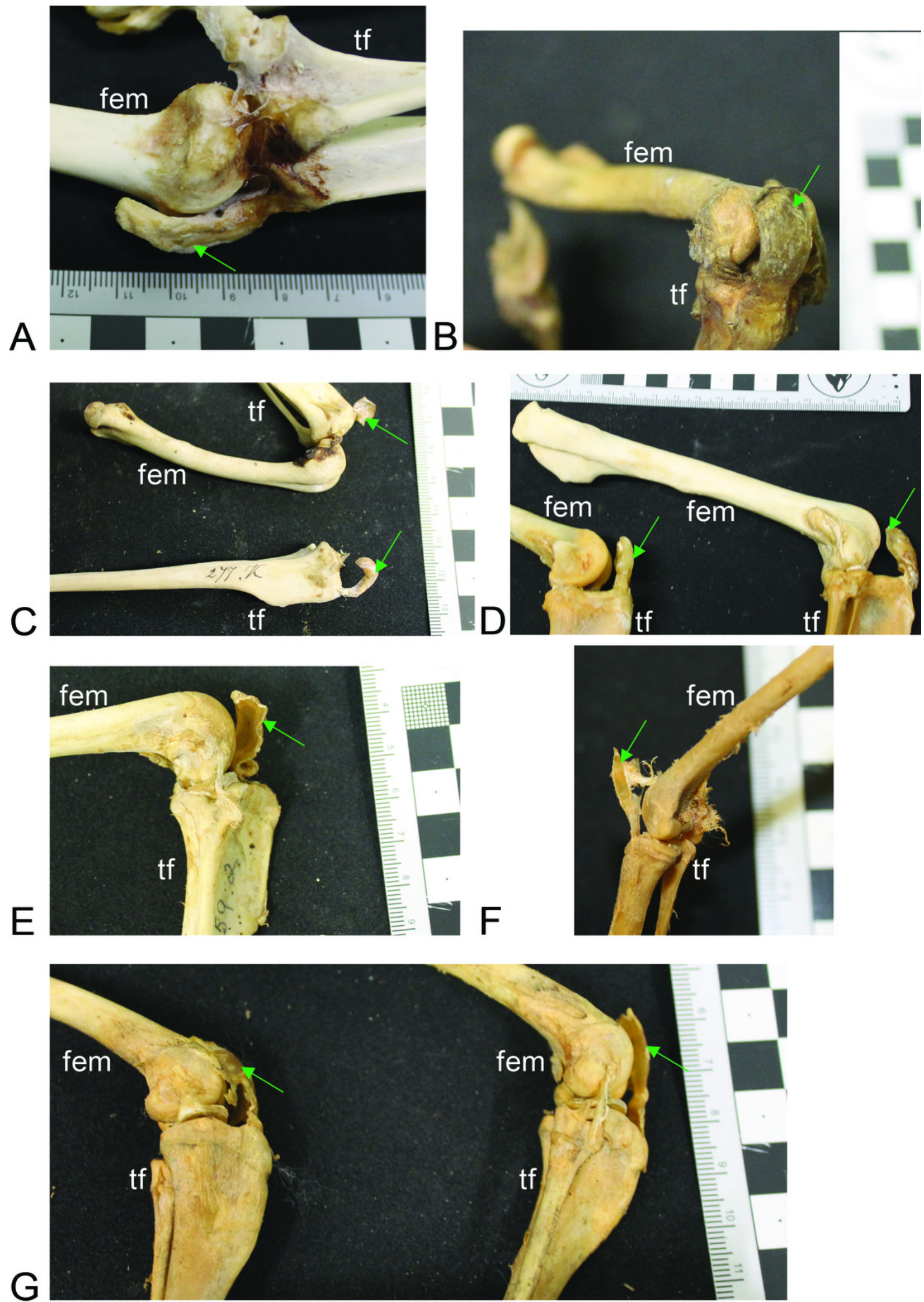


\section{Figure 5}

Patelloids in a specimen of the recently extinct thylacine (Supplementary Text S1: Tables S1,S5).

(A-D): Thylacinus cynocephalus (UMZC display specimen A6.7-1) with patelloids in situ. (A) Photograph of left knee joint in cranial view showing patelloid. (B) Oblique left side view of left and right hindlimbs showing bones and patelloids. (C,D) Slices of left (C) and right (D) knees from micro-CT scans. Note lower density of QF tendon and patelloid (greyer shades) vs. bony tissue (whiter shades) in C and D. Green arrows point to location of patelloid where visible. Red vertical scale bars in $C$ and $D$ are $20 \mathrm{~mm}$. Labels: fem $=$ femur; $t f=$ tibia/fibula. Artefacts (bright white lines through some bones) were caused by ferrous wire. 

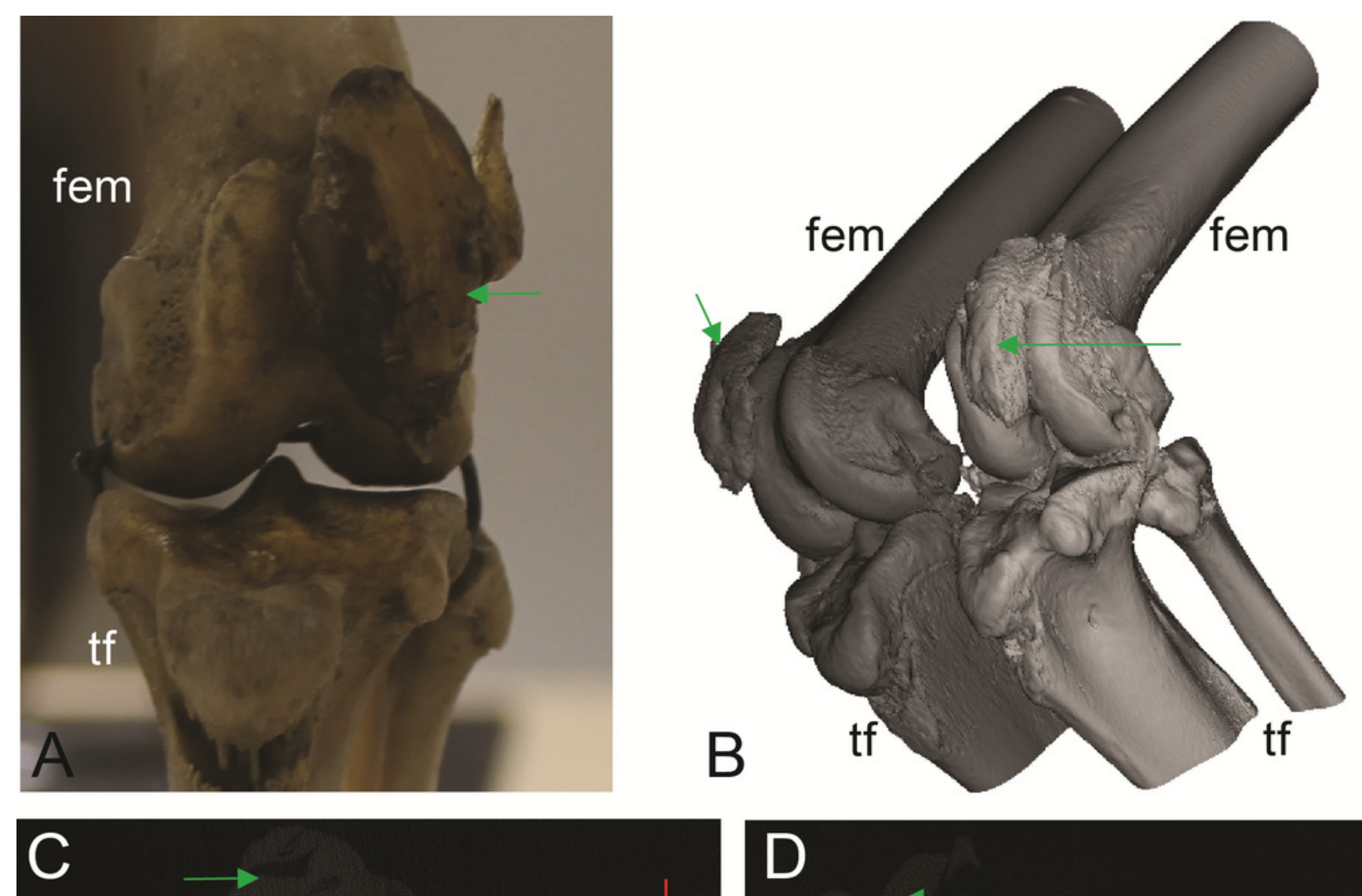

tf

fem

tf 


\section{Figure 6}

Ossified patellae in extant marsupials (Supplementary Text S1: Tables S1,S5).

(A) photograph of Macrotis lagotis (UMZC specimen A7.1-1) bilby, left (top) and right (bottom) hindlimbs. (B) CT scan reconstruction of Notoryctes sp. (UMZC specimen, no number) "marsupial mole", left and right hindlimbs. (C-E) Micro-CT scan slices through knee joints. (C,D): left (C) and right (D) sides of Caenolestes sp. shrew opossum (UMZC specimen A.8.2/3). (E) right side of Isoodon obesulus (UMZC specimen A7.4/5) bandicoot. Green arrows point to location of ossified patella where visible. Note comparably high density of patella vs. other bony tissue (white shades); cf. Figure 3C,E. Labels: "fem" = femur; "Is" = lateral sesamoid; "pes" = pes (hindfoot); "tf" = tibia and fibula. Red scale bars in B-E are, respectively, 12, 2, 2 and $7 \mathrm{~mm}$. 

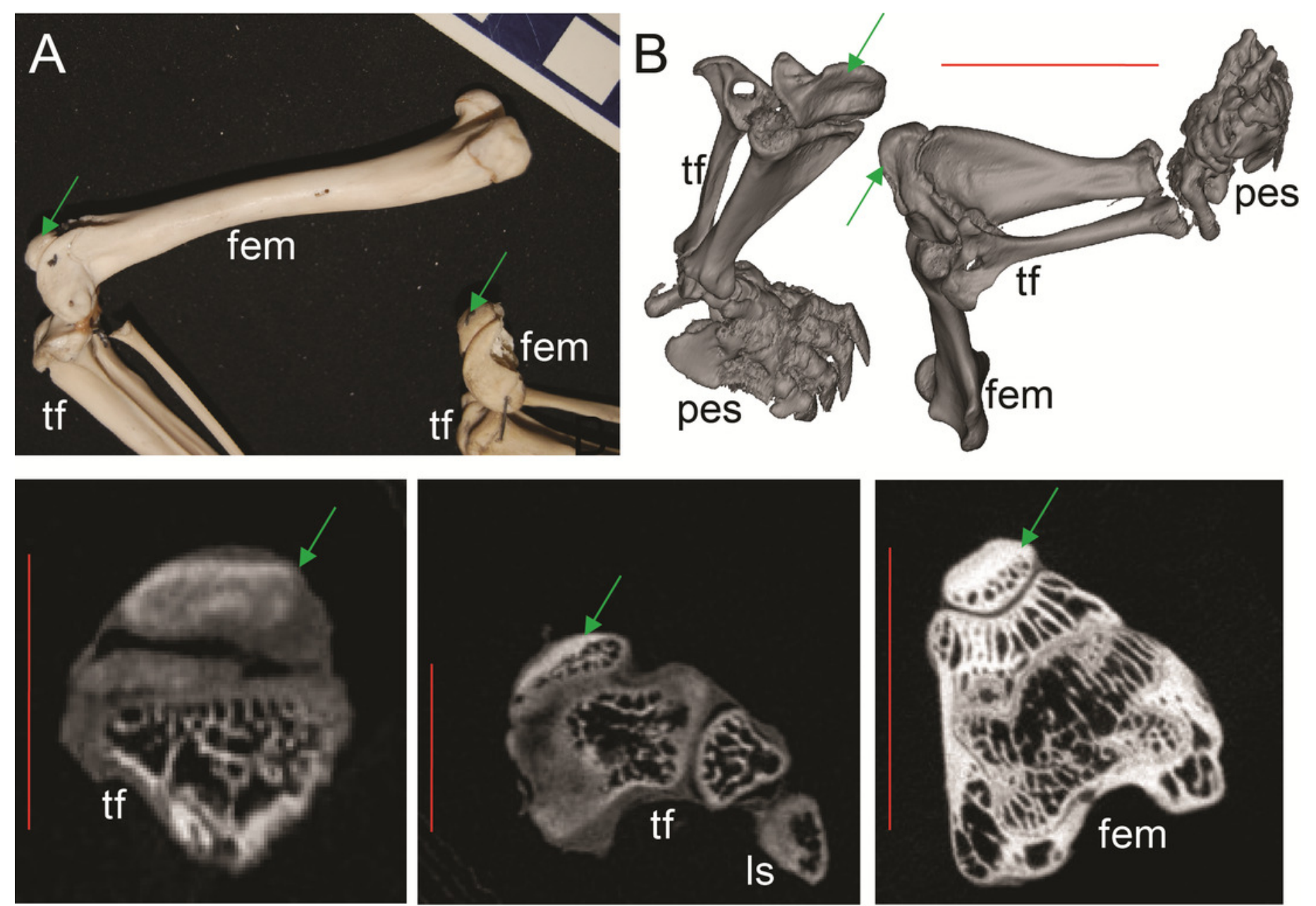

\section{C}

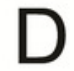

E 


\section{Figure 7}

Example radiographs of skeletal specimens from NHMUK (Supplementary Text S1: Tables S2,S6).

Marker pin has central width of $0.52 \mathrm{~mm}$. (A) Didelphis marsupialis; specimen NHMUK 1959.11.10.1 (opossum); cranio-caudal view; no patellar ossification. (B) Vombatus ursinus; specimen NHMUK 1964.6.29.1 (wombat); medio-lateral view; no patellar ossification.

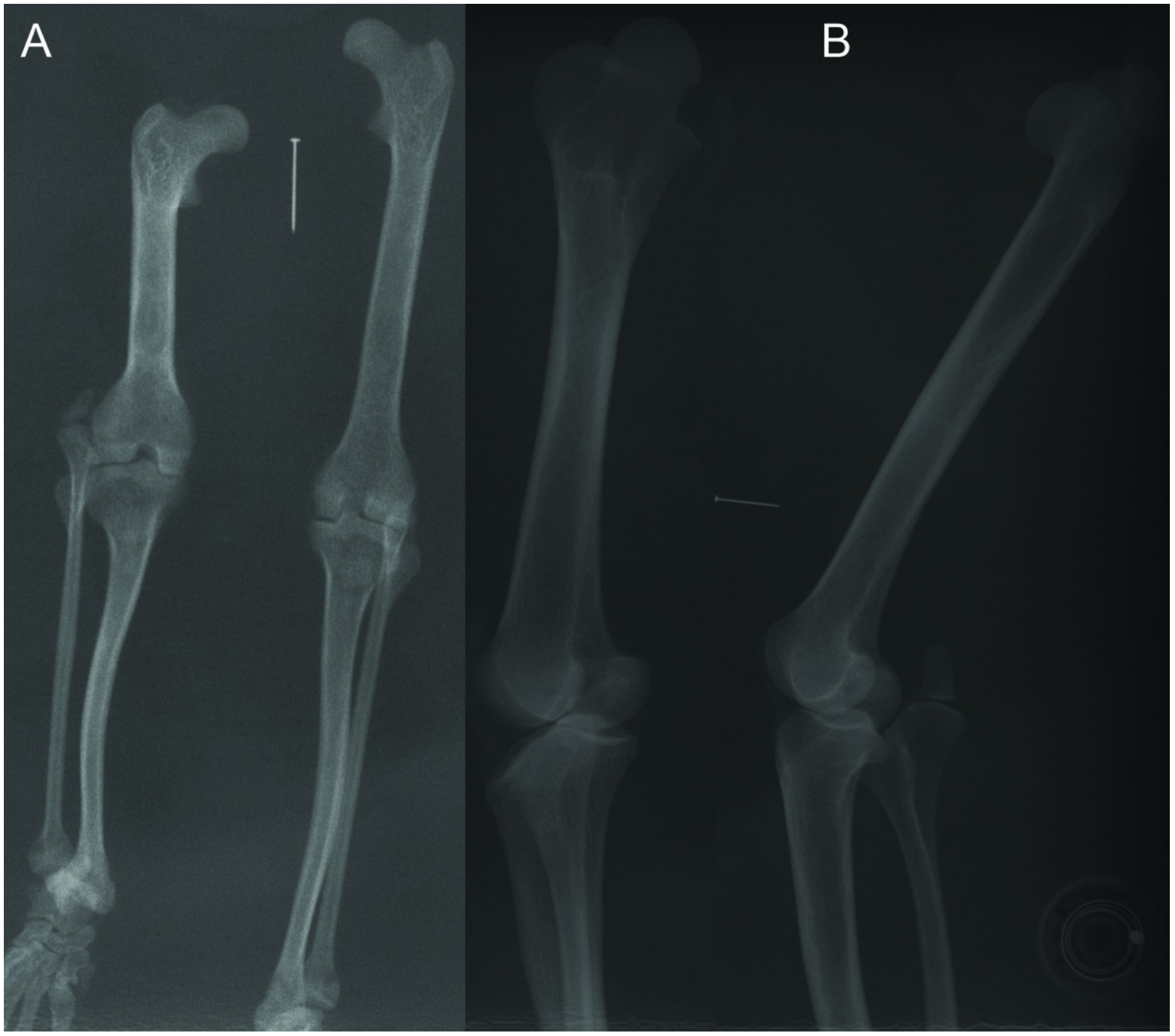




\section{Figure 8}

CT scan revealing presence of ossified tissue in the QF tendon of specimen 1: a recently deceased Macropus rufogriseus (Supplementary Text S1: Table S3), Bennett's wallaby.

$(A, B)$ : 3D reconstructions of femur region from CT scans (bone density only), with medial view in $A$ and cranial view in $B$; $(C, D)$ : longitudinal (C) and mediolateral (D) sections from micro-CT scans. Red vertical scale bars in C and D are $40 \mathrm{~mm}$. Green arrow indicates position of ossified patella. Artefacts through femoral epiphyses were caused by ferrous wire.

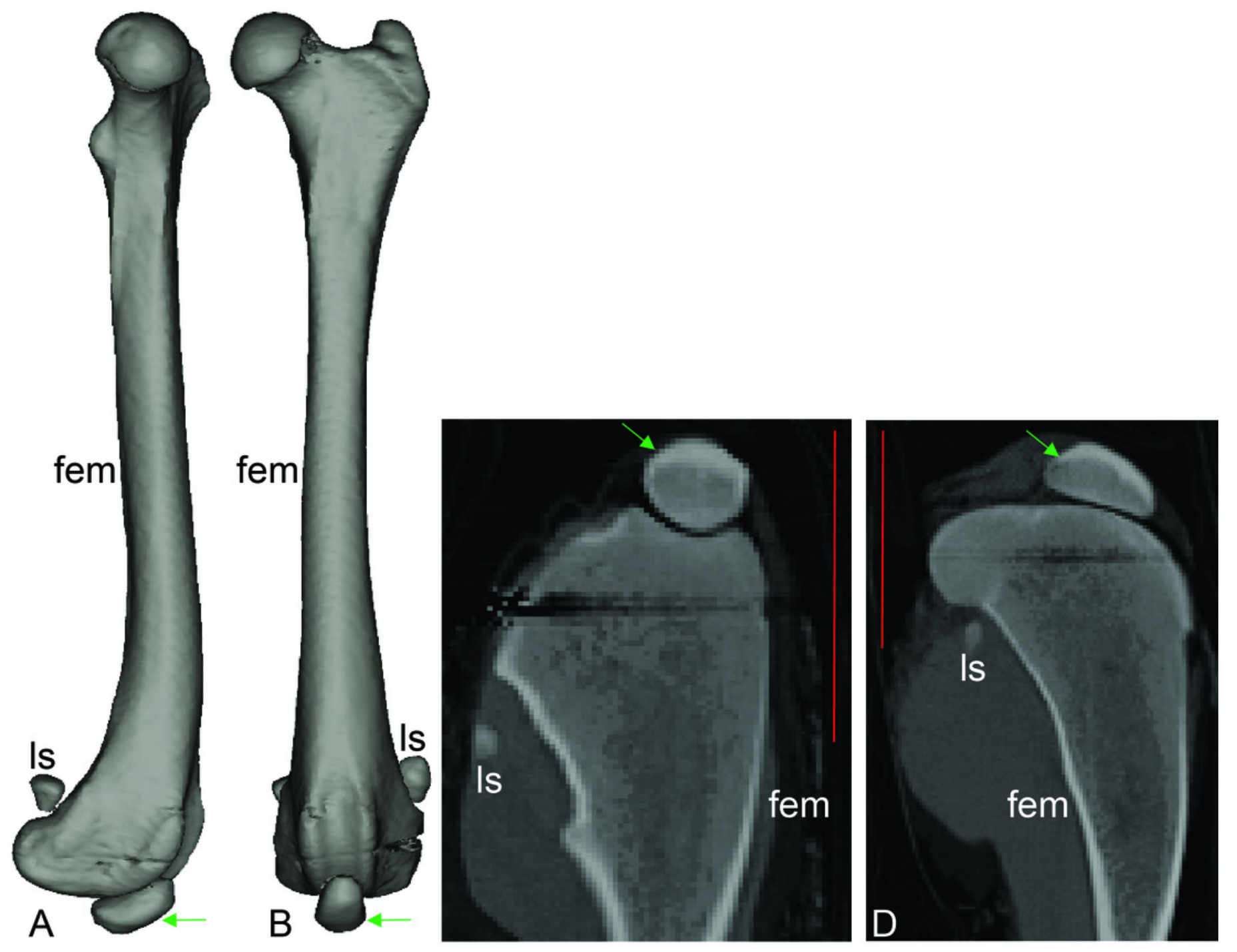




\section{Figure 9}

Photograph of the ossified patella, harvested from specimen 1 of Macropus rufogriseus, following decalcification and cutting.

Not to scale. Labels: FAT = infrapatellar fat pad; PAT = patella main region; QF $=$ QF tendon.

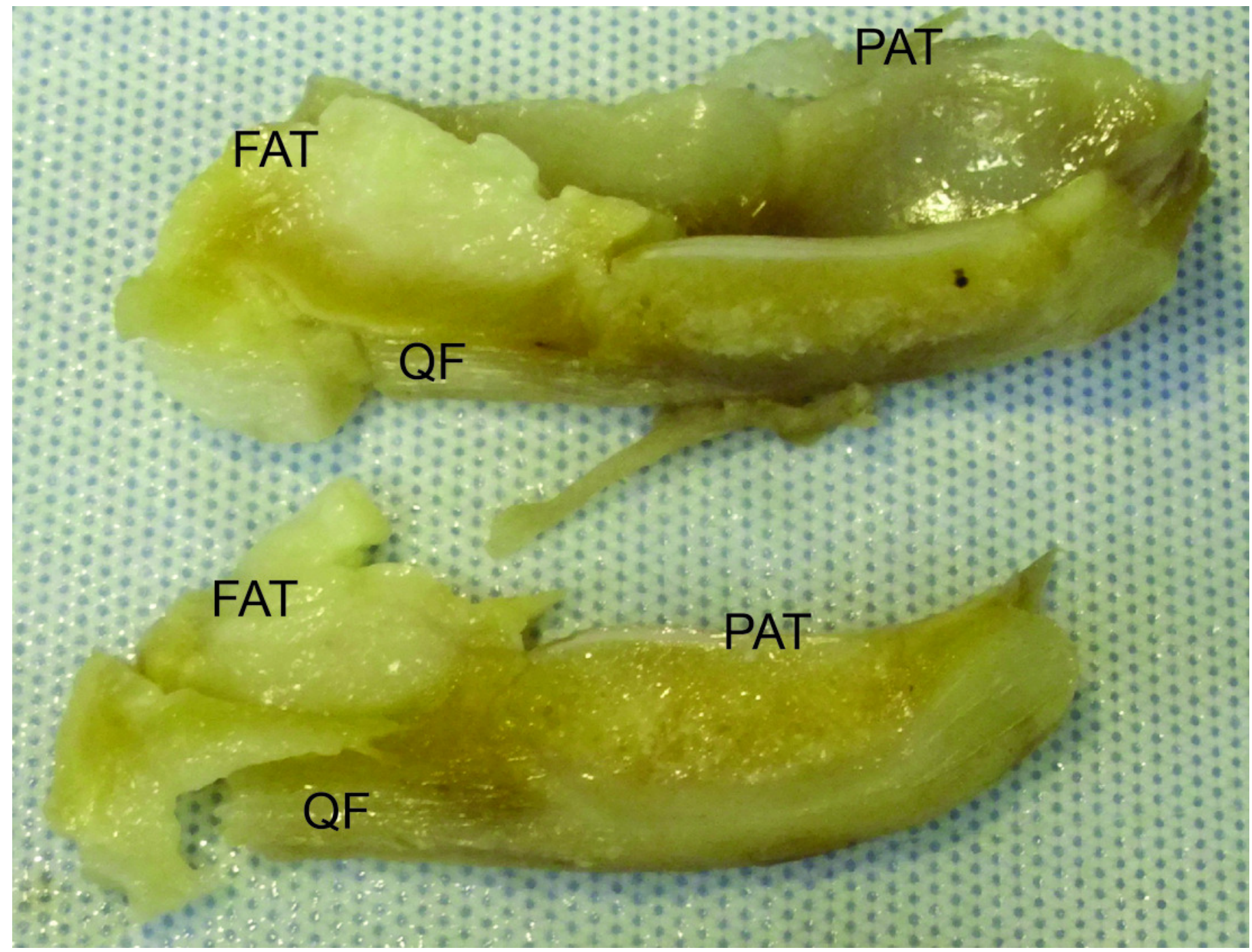


Figure 10

Stained sections from the right and left QF tendons of specimen 1, a Macropus rufogriseus.

One section is illustrated for each stain used. Details of tissues highlighted by each stain are indicated on the left of the figure. An ossified patella is demonstrated in the left tendon (A, C, $E, G)$, while a fibrocartilage patelloid is in the right tendon $(B, D, F, H)$.

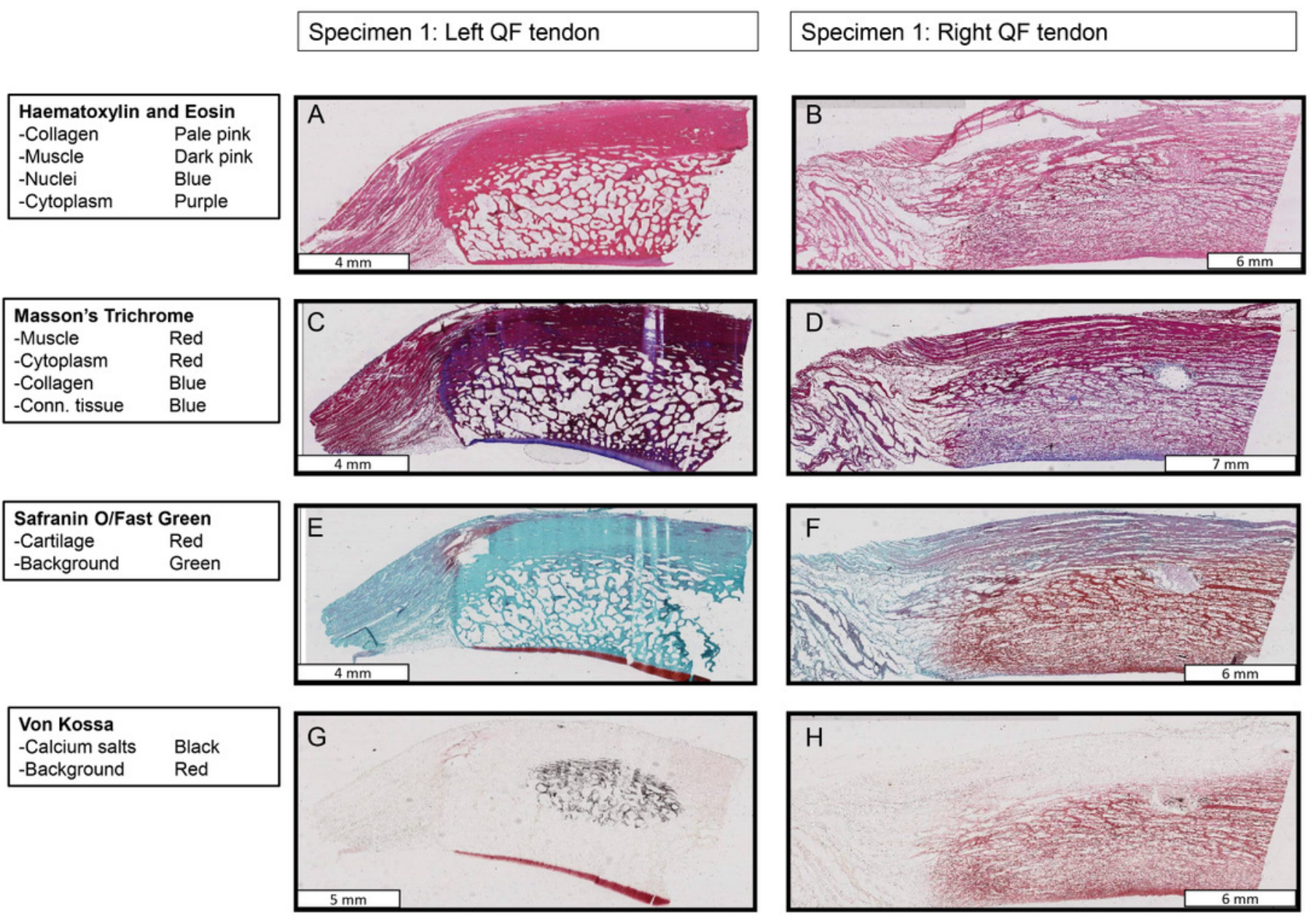




\section{Figure 11}

Higher-magnification of two stained sections of QF tendons from Figure 10, Macropus rufogriseus specimen 1 .

Relevant tissues and orientations are indicated. (A): Haematoxylin \& Eosin stained section of the ossified patella from the left QF tendon. Inset: detail of tendon-bone junction (enthesis); proximal edge of the patella. (B): Safranin O/Fast Green stained section of the fibrocartilage patelloid from the right QF tendon, in higher-magnification view.

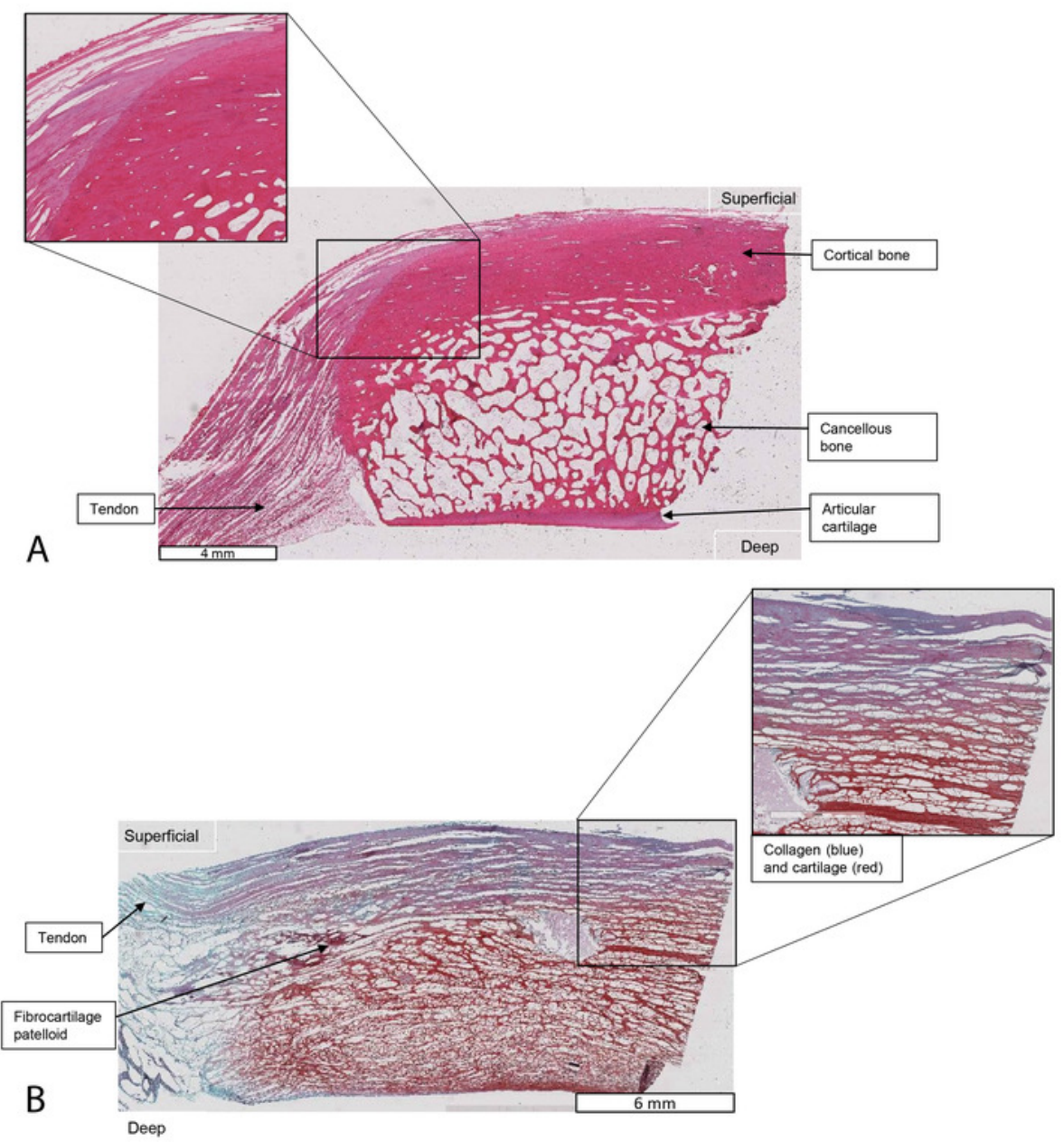




\section{Figure 12}

Ancestral state reconstruction for the patella in marsupial mammals.

Branch colours indicate reconstructed states according to (unordered) maximum parsimony, whilst circles at nodes indicate percentage values of ancestral states according to maximum likelihood (Mk1). Likelihood values for major nodes (\#1-6) are magnified beneath the key. Includes only species for which observations were made or literary references found, mapped onto the original tree used. See Results for interpretations. 
KEY

OAbsent patella

OFibrocartilage patella

Ossified patella

ONo data

Likelihood values:

1. (C)

2. 6

3. 6

4.
5. $\mathbf{O}$
6.

4.
5. $\mathbf{O}$
6.

4.
5. $\mathbf{O}$
6.

0 Petaurus breviceps 0

Petaurus australis

Dactylopsila trivirgata 0

Petauroides volanso

Pseudocheirus peregrinus

Tarsipes rostratus -

Tarsipes rostratus
Acrobates pygmaeus o
Distoechurus pennatuso

Macropus rufus 0

Macropus robustuso

Macropus eugenii

acropus agiliso

acropus dorsaliso

Macropus fuliginosus o

Macropus giganteuso

Macropus rufogriseu

Wallabia bicolor o

Setonix brachyuruso.

Petrogale penicillata

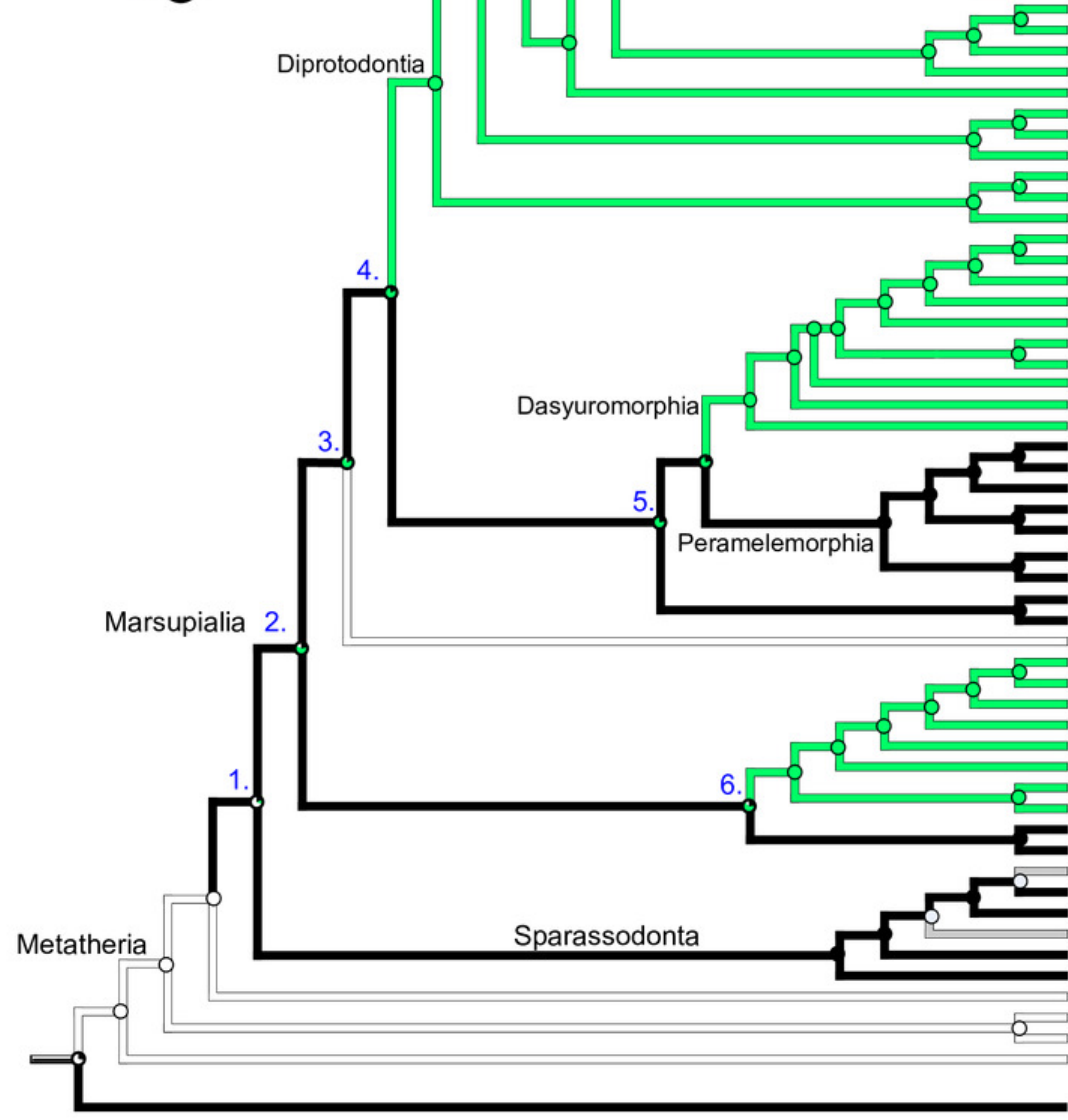

Petrogale persatao

Petrogale persephone

Dendrolagus dorianus

Petauridae \&

Pseudocheiridae

\& Tarsipedidae

Acrobatidae

Macropodidae

(

Thylogalo stigmatica

Thylogale billardieriio

Onychogalea fraenata

Dorcopsis veterum o

Dorcopsis muellerio

Bettongia penicillata =

Bettongia lesueur O

Aepyprymnus rufescenso

Potorous tridactylus o

Hypsiprymnodon moschatuso .

richosurus vulpecula

Burramys parvuso

Vombatus ursinus 0

Lasiorhinus latifrons o

Phascolarctos cinereuso

Dasyurus maculatus 0

Dasyurus viverrinuso

Dasyurus hallucatus

Sarcophilus harrisii o

Antechinus lapoinafa 0

Sminthopsis crassicaudata

Ayrmecobius fasciatuso

Thylacinus cynosciatus 0

Perameles nasuta =

Perameles gunnii

Perameles bougainville

soodon auratus

Isoodon obesulus

Macrotis lagotis

Macrotis leucura

Notoryctes typhlops

Notoryctes caurinus

Dromiciops gliroides

Didelphis marsupialis

Didelphis albiventris

Didelphis virginiana

Lutreolina crassicaudata 0

Chironectes minimus 0

Metachirus nudicaudatus

Caluromys lanatus

Caluromys philanderO

Caenolestes fuliginosus -

Potoridae

Phalangeridae

= Burramyidae

- Vombatidae

$=$ Phascolarctidae

Dasyuridae

$=$

Peramelidae

Thylacomyidae

Notoryctidae

$=$ Microbiotheriidae

Didelphidae

a

Prothylacinus

Lycopsis O

Callistoe $\bullet$

Sipalocyon -

Thetotherium

Maylestes O

Asiatherium

Eutheria 


\section{Figure 13}

Dissections of an adult male koala; right hindlimb in cranial view of knee.

(A) Specimen skinned with superficial surface of patelloid exposed and QF muscle/tendon partially reflected. (B) QF muscle/tendon fully reflected below distal femur to expose deep surface of patelloid. The specimen was obtained by Monash University from Museums Victoria (registered as MUPC5), from the Victorian Department of Sustainability and Environment cull program of 7 March 2014, Bimbi Park, Cape Otway, Victoria, Australia (38 5002 S, 1433047 E) under Flora and Fauna Permit number 10007596. Photos and description courtesy of Hazel Richards. Not to scale. Labels: fem = (distal end of) femur; pat = patelloid; QF $=$ QF muscles/tendon.

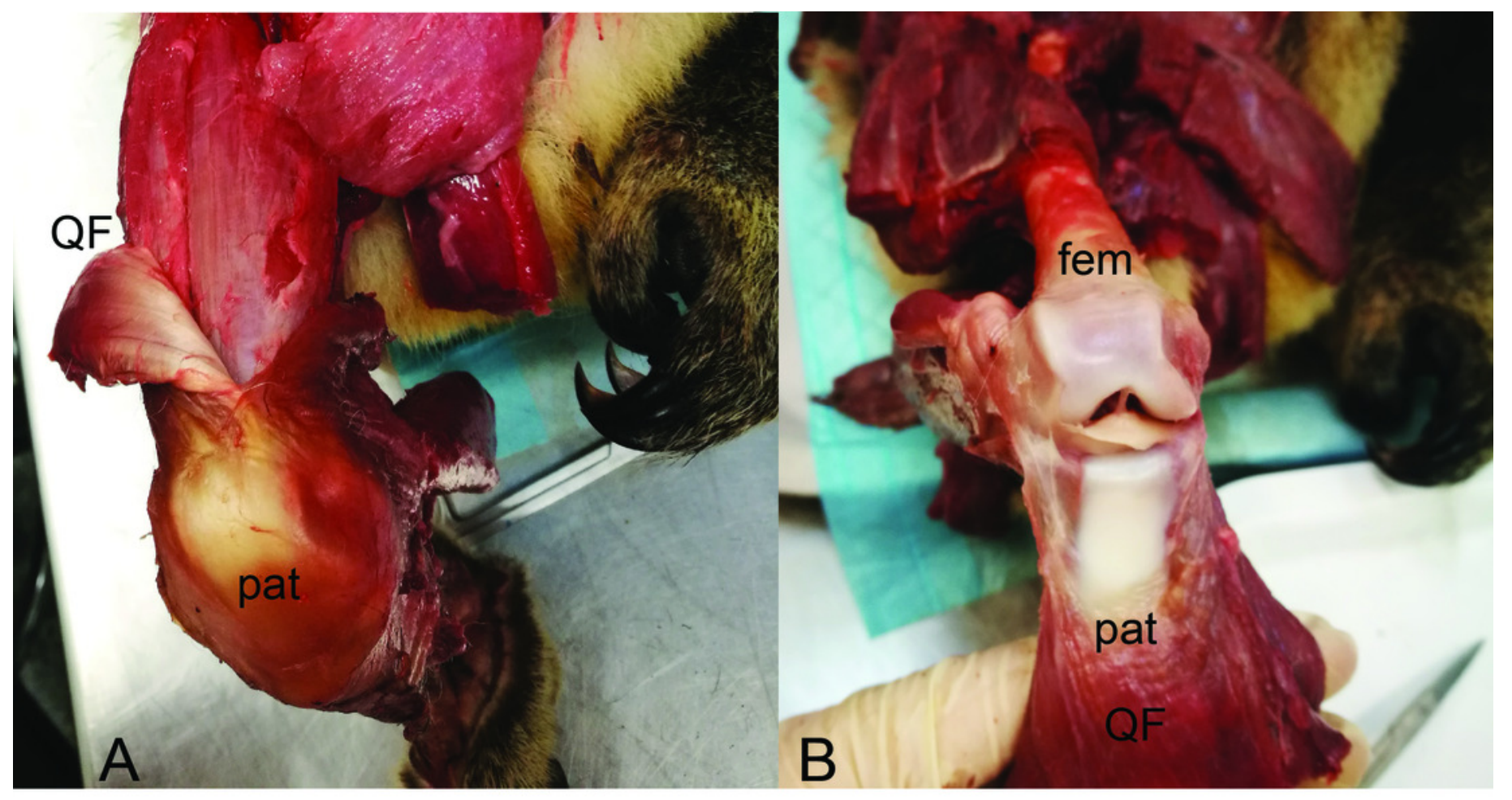

